\title{
QUANTUM GROUPS AND NONCOMMUTATIVE GEOMETRY
}

\author{
Shahn Majid \\ School of Mathematical Sciences, Queen Mary and Westfield College \\ University of London, Mile End Rd, London E1 4NS, UK
}

November, 1999

\begin{abstract}
Quantum groups emerged in the latter quarter of the 20th century as, on the one hand, a deep and natural generalisation of symmetry groups for certain integrable systems, and on the other as part of a generalisation of geometry itself powerful enough to make sense in the quantum domain. Just as the last century saw the birth of classical geometry, so the present century sees at its end the birth of this quantum or noncommutative geometry, both as an elegant mathematical reality and in the form of the first theoretical predictions for Planck-scale physics via ongoing astronomical measurements. Noncommutativity of spacetime, in particular, amounts to a postulated new force or physical effect called cogravity.
\end{abstract}

\section{Introduction}

Now that quantum groups and their associated quantum geometry have been around for more than a decade, it is surely time to take stock. Where did quantum groups come from, what have they achieved and where are they going? This article, which is addressed to non-specialists (but should also be interesting for experts) tries to answer this on two levels. First of all on the level of quantum groups themselves as mathematical tools and building blocks for physical models. And, equally importantly, quantum groups and their associated noncommutative geometry in terms of their overall significance for mathematics and theoretical physics, i.e., at a more conceptual level. Obviously this latter aspect will be very much my own perspective, which is that of a theoretical physicist who came to quantum groups a decade ago as a tool to unify quantum theory and gravity in an algebraic approach to Planck scale physics. This is in fact only one of the two main origins in physics of quantum groups; the other being integrable systems, which I will try to cover as well. Let me also say that noncommutative geometry has other approaches, notably the one of A. Connes coming out of operator theory. I will say something about this too, although, until recently, this has largely been a somewhat different approach.

We start with the conceptual significance for theoretical physics. It seems clear to me that future generations looking back on the 20th century will regard the discovery of quantum mechanics in the 1920 s, i.e. the idea to replace the coordinates $x, p$ of classical mechanics by 
noncommuting operators $\mathbf{x}, \mathbf{p}$, as one of its greatest achievements in our understanding of Nature, matched in its significance only by the unification of space and time as a theory of gravity. But whereas the latter was well-founded in the classical geometry of Newton, Gauss, Riemann and Poincaré, quantum theory was something much more radical and mysterious. Exactly which variables in the classical theory should correspond to operators? They are local coordinates on phase space but how does the global geometry of the classical theory look in the quantum theory, what does it fully correspond to? The problem for most of this century was that the required mathematical structures to which the classical geometry might correspond had not been invented and such questions could not be answered.

As I hope to convince the reader, quantum groups and their associated noncommutative geometry have led in the last decades of the 20th century to the first definitive answers to this kind of question. There has in fact emerged a more or less systematic generalisation of geometry every bit as radical as the step from Euclidean to non-Euclidean, and powerful enough not to break down in the quantum domain. I do doubt very much that what we know today will be the final formulation, but it is a definitive step in a right and necessary direction and a turning point in the future development of mathematical and theoretical physics. For example, any attempt to build a theory of quantum gravity with classical starting point a smooth manifold this includes loop-variable quantum gravity, string theory and quantum cosmology, is necessarily misguided except as some kind of effective approximation: smooth manifolds should come out of the algebraic structure of the quantum theory and not be a starting point for the latter. There is no evidence that the real world is any kind of smooth continuum manifold except as a macroscopic approximation and every reason to think that it is fundamentally not. I therefore doubt that any one of the above could be a 'theory everything' until it becomes an entirely algebraic theory founded in noncommutative geometry of some kind or other. Of course, this is my personal view.

At any rate, I do not think that the fundamental importance of noncommutative geometry can be overestimated. First of all, anyone who does quantum theory is doing noncommutative geometry whether wanting to admit it or not, namely noncommutative geometry of the phase space. Less obvious but also true, we will see in Section II that if the position space is curved then the momentum space is by itself intrinsically noncommutative. If one gets this far then it is also natural that the position space or spacetime by itself could be noncommutative, which would correspond to a curved or nonAbelian momentum group. This is one of the bolder predictions coming out of noncommutative geometry. It has the simple physical interpretation as what I call cogravity, i.e. curvature or 'gravity' in momentum space. As such it is independent of i.e. dual to curvature or gravity in spacetime and would appear as a quite different and new physical effect. Theoretically cogravity can, for example, be detected as energy-dependence of the speed 


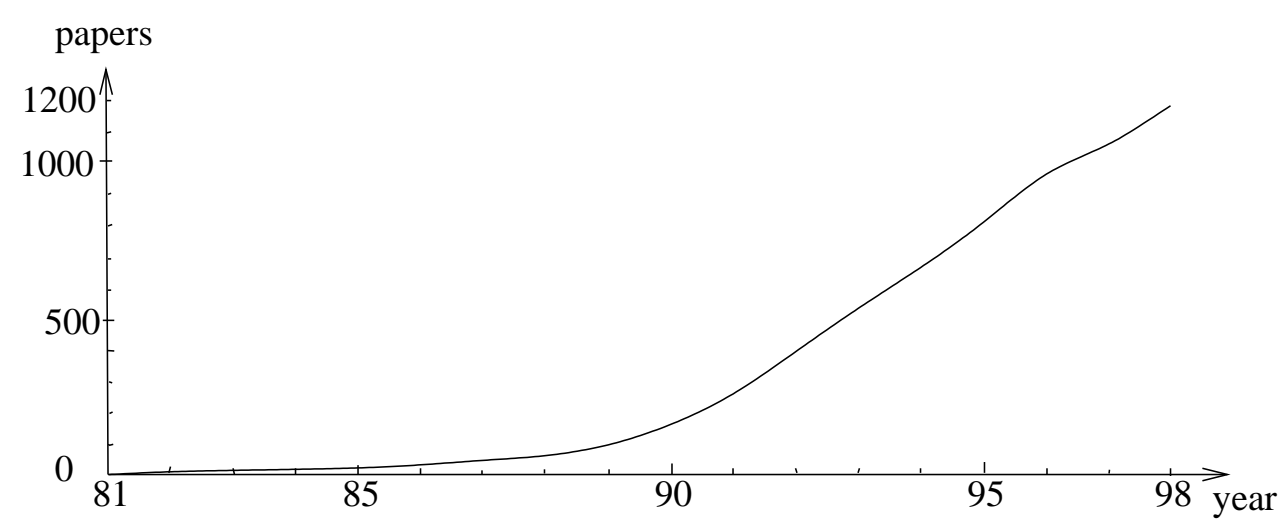

Figure 1: Growth of research papers on quantum groups

of light. Moreover, even if cogravity was very weak, of the order of a Planck-scale effect, it could still in principle be detected by astronomical measurements at a cosmological level. Therefore, just in time for the new millennium, we have the possibility of an entirely new physical effect in Nature coming from fresh and conceptually sound new mathematics.

Where quantum groups precisely come into this is as follows. Just as Lie groups and their associated homogeneous spaces provided definitive examples of classical differential geometry even before Riemann formulated their intrinsic structure as a theory of manifolds, so quantum groups and their associated quantum homogeneous spaces, quantum planes etc., provide large (i.e. infinite) classes of examples of proven mathematical and physical worth and clear geometrical content on which to build and develop noncommutative differential geometry. They are noncommutative spaces in the sense that they have generators or 'coordinates' like the noncommuting operators $\mathbf{x}, \mathbf{p}$ in quantum mechanics but with a much richer and more geometric algebraic structure than the Heisenberg or CCR algebra. In particular, I do not believe that one can build a theory of noncommutative differential geometry based on only one example such as the Heisenberg algebra or its variants (however fascinating) such as the much-studied noncommutative torus. One needs many more 'sample points' in the form of natural and varied examples to obtain a valid general theory. By contrast, if one does a search of BIDS one finds, see Figure 1, vast numbers of papers in which the rich structure and applications of quantum groups are explored and justified in their own right (data complied from BIDS: published papers since 1981 with title or abstract containing 'quantum group*', 'Hopf alg*','noncommutative geom*', 'braided categ*', 'braided group*', 'braided Hopf*'.) This is the significance of quantum groups. And of course something like them should be needed in a quantum world where there is no evidence for a classical space such as the underlying set of a Lie group.

Finally, it turns out that noncommutative geometry, at least of the type that we shall de- 
scribe, is in many ways cleaner and more straightforward than the special commutative limit. One simply does not need to assume commutativity in most geometrical constructions, including differential calculus and gauge theory. The noncommutative version is often less infinite, differentials are often more regular finite-differences, etc. And noncommutative geometry (unlike classical geometry) can be specialised without effort to discrete spaces or to finite-dimensional algebras. It is simply a powerful and natural generalisation of geometry as we usually know it. So my overall summary and prediction for the next millennium from this point of view is:

- All geometry will be noncommutative (or whatever comes beyond that), with conventional geometry merely a special case.

- The discovery of quantum theory, its correspondence principle (and noncommutative geometry is nothing more than the elaboration of that) will be considered one of the century's greatest achievement in mathematical physics, commensurate with the discovery of classical geometry by Newton some centuries before.

- Quantum groups will be viewed as the first nontrivial class of examples and thereby pointers to the correct structure of this noncommutative geometry.

- Spacetime too (not only phase space) will be known to be noncommutative (cogravity will have been detected).

- At some point a future Einstein will combine the then-standard noncommutative geometrical ideas with some deep philosophical ideas and explain something really fundamental about our physical reality.

In the fun spirit of this article, I will not be above putting down my own thoughts on this last point. These have to do with what I have called for the last decade the Principle of representation-theoretic self-duality [1]. In effect, it amounts to extending the ideas of Born reciprocity, Mach's principle and Fourier theory to the quantum domain. Roughly speaking, quantum gravity should be recast as gravity and cogravity both present and dual to each other and with Einstein's equation appearing as a self-duality condition. The longer-term philosophical implications are a Kantian or Hegelian view of the nature of physical reality, which I propose in Section $\mathrm{V}$ as a new foundation for next millennium.

We now turn to another fundamental side of quantum groups, which is at the heart of their other origin in physics, namely as generalised symmetry groups in exactly solvable lattice models. It leads to diverse applications ranging from knot theory to representation theory to Poisson geometry, all areas that quantum groups have revolutionised. What is really going on here in my opinion is not so much the noncommutative geometry of quantum groups themselves as a different kind of noncommutativity or braid statistics which certain quantum groups induce on 
any objects of which they are a symmetry. The latter is what I have called 'noncommutativity of the second kind' or outer noncommutativity since it not so much a noncommutativity of one algebra as a noncommutative modification of the exchange law or tensor product of any two independent algebras or systems. It is the notion of independence which is really being deformed here. Recall that the other great 'isation' idea in mathematical physics in this century (after 'quantisation') was 'superisation', where everything is $\mathbb{Z}_{2}$-graded and this grading enters into how two independent systems are interchanged. Physics traditionally has a division into bosonic or force particles and fermionic or matter particles according to this grading and exchange behaviour. So certain quantum groups lead to a generalisation of that as braided geometry[2] or a process of braidification. These quantum groups typically have a parameter $q$ and its meaning is a generalisation of the -1 for supersymmetry. This in turn leads to a profound generalisation of conventional (including super) mathematics in the form of a new concept of algebra wherin one 'wires up' algebraic operations much as the wiring in a computer, i.e. outputs of one into inputs of another. Only, this time, the under or over crossings are nontrivial (and generally distinct) operations depending on $q$. These are the so-called 'R-matrices'. Afterwards one has the luxury of both viewing $q$ in this way or expanding it around 1 in terms of a multiple of Planck's constant and calling it a formal 'quantisation' - $q$-deformation actually unifies both 'isation' processes. For example, Lorentz-invariance, by the time it is $q$-deformed[3], induces braid statistics even when particles are initially bosonic. In summary,

- The notion of symmetry or automorphism group is an artifact of classical geometry and in a quantum world should naturally be generalised to something more like a quantum group symmetry.

- Quantum symmetry groups induce braid statistics on the systems on which they act. In particular, the notion of bose-fermi statistics or the division into force and matter particles is an artifact of classical geometry.

- Quantisation and the departure from bosonic statistics are two limits of the same phenomenon of braided geometry.

Again, there are plenty of concrete models in solid state physics already known with quantum group symmetry. The symmetry is useful and can be viewed (albeit with hindsight) as the origin of the exact solvability of these models.

These two points of view, the noncommutative geometrical and the generalised symmetry, are to date the two main sources of quantum groups. One has correspondingly two main flavours or types of quantum groups which really allowed the theory to take off. Both were introduced at the mid 1980s although the latter have been more extensively studied in terms of applications 
to date. They include the deformations

$$
U_{q}(\mathfrak{g})
$$

of the enveloping algebra $U(\mathfrak{g})$ of every complex semisimple Lie algebra $\mathfrak{g}$ [四河]. These have as many generators as the usual ones of the Lie algebra but modified relations and, additionally, a structure called the 'coproduct'. The general class here is that of quasitriangular quantum groups. They arose as generalised symmetries in certain lattice models but are also visible in the continuum limit quantum field theories (such as the Wess-Zumino-Novikov-Witten model on the Lie group $G$ with Lie algebra $\mathfrak{g})$. The coordinate algebras of these quantum groups are further quantum groups $\mathbb{C}_{q}[G]$ deforming the commutative algebra of coordinate functions on $G$. There is again a coproduct, this time expressing the group law or matrix multiplication. Meanwhile, the type coming out of Planck scale physics [6] are the bicrossproduct quantum groups

$$
\mathbb{C}[M] \bowtie U(\mathfrak{g})
$$

associated to the factorisation of a Lie group $X$ into Lie subgroups, $X=G M$. Here the ingredients are the conventional enveloping algebra $U(\mathfrak{g})$ and the commutative coordinate algebra $\mathbb{C}[M]$. The factorisation is encoded in an action and coaction of one on the other to make a semidirect product and coproduct $\bowtie$. These quantum arose at about the same time but quite independently of the $U_{q}(\mathfrak{g})$, as the quantum algebras of observables of certain quantum spaces. Namely it turns out that $G$ acts on the set $M$ (and vice-versa) and the quantisation of those orbits are these quantum groups. This means that they are literally noncommutative phase spaces of honest quantum systems. In particular, every complex semisimple $\mathfrak{g}$ has an associated complexification and its Lie group factorises $G_{\mathbb{C}}=G G^{\star}$ (the classical Iwasawa decomposition) so there is an example

$$
\mathbb{C}\left[G^{\star}\right] \bowtie U(\mathfrak{g})
$$

built from just the same data as for $U_{q}(\mathfrak{g})$. In fact the Iwasawa decomposition can be understood in Poisson-Lie terms with $\mathfrak{g}^{\star}$ the classical 'Yang-Baxter dual' of $\mathfrak{g}$. In spite of this, there is, even after a decade of development, no direct connection between the two quantum groups:

$$
\begin{gathered}
\mathfrak{g} \\
\swarrow \quad \searrow \\
U_{q}(\mathfrak{g}) \quad \leftarrow \rightarrow \mathbb{C}\left[G^{\star}\right] \bowtie U(\mathfrak{g}) .
\end{gathered}
$$

They are both 'exponentiations' of the same classical data but apparently of completely different type (this remains a mystery to date.) 


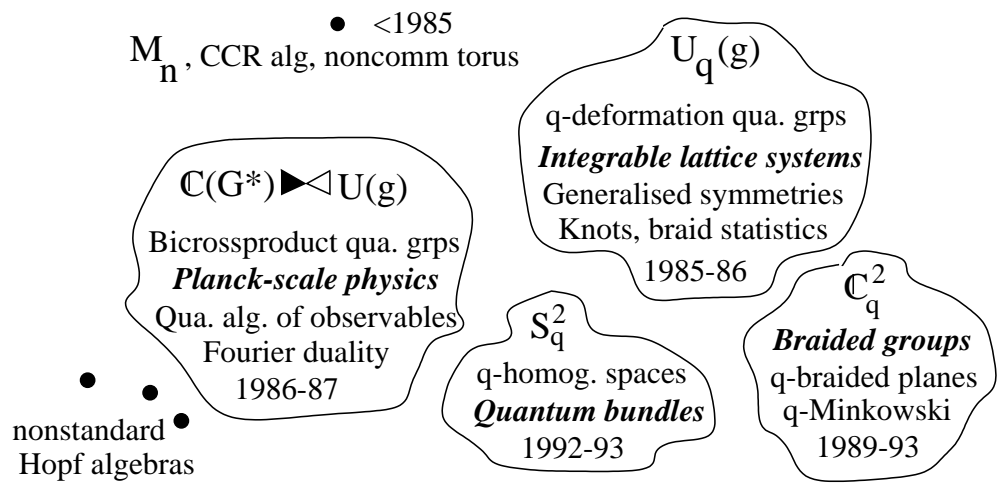

Figure 2: The landscape of noncommutative geometry today

Associated to these two flavours of quantum groups there are corresponding homogeneous spaces such as quantum spheres, quantum spacetimes, etc. Thus, of the first type there is a $q$-Minkowski space introduced in [7] as a $q$-Lorentz covariant algebra, and independently about a year later in [8] as $2 \times 2$ braided hermitian matrices. It is characterised by

$$
\left[x_{i}, t\right]=0, \quad\left[x_{i}, x_{j}\right] \neq 0
$$

Meanwhile, of the second type there is a noncommutative $\lambda$-Minkowski space with

$$
\left[x_{i}, t\right]=\lambda x_{i}, \quad\left[x_{i}, x_{j}\right]=0
$$

which is the one that provides the first known predictions testable by astronomical measurements (by gamma-ray bursts of cosmological origin[9]). This kind of algebra was proposed as spacetime in [10] and in the 4-dimensional case it was shown in [11] to be covariant under a Poincaré quantum group of bicrossproduct form. These are clearly in sharp contrast.

There are of course many more objects than these. $q$-spheres, $q$-planes etc. In Section IV we turn to the notion of 'quantum manifold' that is emerging from all these examples. Riemann was able to formulate the notion of Riemannian manifold as a way to capture known examples like spheres and tori but broad enough to formulate general equations for the intrinsic structure of space itself (or after Einstein, space-time). We are at a similar point now and what this 'quantum groups approach to noncommutative geometry' is is more or less taking shape. It has the same degree of 'flabbiness' as Riemannian geometry (it is not tied to specific integrable systems etc.) while at the same time it includes the 'zoo' of already known naturally occurring examples, mostly linked to quantum groups. Such things as Ricci tensor and Einstein's equation are not yet understood from this approach, however, so I would not say it is the last word.

This approach is in fairly sharp contrast to 'traditional' noncommutative geometry as it was done before the emergence of quantum groups. That theory was developed by mathematicians 
and mathematical physicists also coming from quantum mechanics but being concerned more with topological completions and Hilbert spaces. Certainly a beautiful theory of von-Neumann and $C^{*}$ algebras emerged as an analogue of point-set topology. Some general methods such as cyclic cohomology were also developed in the 1970s, with remarkable applications throughout mathematics [12]. However, for concrete examples with actual noncommutative differential geometry one usually turned either to an actual manifold as input datum or to the Weyl algebra (or noncommutative torus) defined by relations

$$
v u=e^{2 \pi \imath \theta} u v .
$$

This in turn is basically the usual CCR or Heisenberg algebra

$$
[x, p]=\imath \hbar
$$

in exponentiated form. And at an algebraic level (i.e. until one considers the precise $C^{*}$ algebra completion) this is basically the usual algebra $B(\mathcal{H})$ of operators on a Hilbert space as in quantum mechanics. Or at roots of unity it is $M_{n}(\mathbb{C})$ the algebra of $n \times n$ matrices. So at some level these are all basically one example. Unfortunately many of the tricks one can pull for this kind of example are special to it and not a foundation for noncommutative differential geometry of the type we need. For example, to do gauge theory Connes and M. Rieffel 13] used derivations for two independent vector fields on the torus. The formulation of 'vector field' as a derivation of the coordinate algebra is what I would call the traditional approach to noncommutative geometry. For quantum groups such as $\mathbb{C}_{q}[G]$ one simply does not have those derivations (rather, they are in general braided derivations). Similarly, in the traditional approach one defines a 'vector bundle' as a finitely-generated projective module without any of the infrastructure of differential geometry such as a principal bundle to which the vector bundle might be associated, etc. All of that could not emerge until quantum groups arrived (one clearly should take a quantum group as fiber). This is how the quantum groups approach differs from the work of Connes, Rieffel, Madore and others. It is also worth noting that string theorists have recently woken up to the need for a noncommutative spacetime but, so far at least, have still considered only this 'traditional' Heisenberg-type algebra. In the last year or two there has been some success in merging these approaches, however; a trend surely to be continued. By now both approaches have a notion of 'noncommutative manifold' which appear somewhat different but which have as point of contact the Dirac operator.

Preliminaries. A full text on quantum groups is [14]. To be self-contained we provide here a quick definition. Later on we will see many examples and various justifications for this concept. Thus, a quantum group or Hopf algebra is

- A unital algebra $H, 1$ over the field $\mathbb{C}$ (say) 
- A coproduct $\Delta: H \rightarrow H \otimes H$ and counit $\epsilon: H \rightarrow \mathbb{C}$ forming a coalgebra, with $\Delta, \epsilon$ algebra homomorphisms.

- An antipode $S: H \rightarrow H \operatorname{such}$ that $\cdot(S \otimes$ id $) \Delta=1 \epsilon=\cdot($ id $\otimes S) \Delta$.

Here a coalgebra is just like an algebra but with the axioms written as maps and arrows on the maps reversed. Thus the coassociativity and counity axioms are

$$
(\Delta \otimes \mathrm{id}) \Delta=(\mathrm{id} \otimes \Delta) \Delta, \quad(\epsilon \otimes \mathrm{id}) \Delta=(\mathrm{id} \otimes \epsilon) \Delta=\mathrm{id} .
$$

The antipode plays a role that generalises the concept of group inversion. Other than that the only new mathematical structure that the reader has to contend with is the coproduct $\Delta$ and its associated counit. There are several ways of thinking about the meaning of this depending on our point of view. If the quantum group is like the enveloping algebra $U(\mathfrak{g})$ generated by a Lie algebra $\mathfrak{g}$, one should think of $\Delta$ as providing the rule by which actions extend to tensor products. Thus, $U(\mathfrak{g})$ is trivially a Hopf algebra with

$$
\Delta \xi=\xi \otimes 1+1 \otimes \xi, \quad \forall \xi \in \mathfrak{g},
$$

which says that when a Lie algebra element $\xi$ acts on tensor products it does so by $\xi$ in the first factor and then $\xi$ in the second factor. Similarly it says that when a Lie algebra acts on an algebra it does so as a derivation. On the other hand, if the quantum group is like a coordinate algebra $\mathbb{C}[G]$ then $\Delta$ expresses the group multiplication and $\epsilon$ the group identity element $e$. Thus, if $f \in \mathbb{C}[G]$ the coalgebra is

$$
(\Delta f)(g, h)=f(g h), \quad \forall g, h \in G \quad \epsilon f=f(e)
$$

at least for suitable $f$ (or with suitable topological completions). In other words it expresses the group product $G \times G \rightarrow G$ by a map in the other direction in terms of coordinate algebras. From yet another point of view $\Delta$ simply makes the dual $H^{*}$ also into an algebra. So a Hopf algebra is basically an algebra such that $H^{*}$ is also an algebra, in a compatible way, which makes the axioms 'self-dual'. For every finite-dimensional $H$ there is a dual $H^{*}$. Similarly in the infinite-dimensional case. It said that in the Roman empire, 'all roads led to Rome'. It is remarkable that several different ideas for generalising groups all led to the same axioms. The axioms themselves were first introduced (actually in a super context) by H. Hopf in 1947 in his study of group cohomology but the subject only came into its own in the mid 1980s with the arrival from mathematical physics of the large classes of examples (as above) that are neither like $U(\mathfrak{g})$ nor like $\mathbb{C}[G]$, i.e. going truly beyond Lie theory or algebraic group theory.

Acknowledgements. An announcement of this article appears in a short millennium article 15 and a version more focused on the meaning for Planck scale physics in 16]. 


\section{Quantum groups and Planck scale physics}

This section covers quantum groups of the bicrossproduct type coming out of Planck-scale physics [6] and their associated noncommutative geometry. These are certainly less well-developed than the more familiar $U_{q}(\mathfrak{g})$ in terms of their concrete applications; one does not have interesting knot invariants etc. On the other hand, these quantum groups have a clearer physical meaning as models of Planck scale physics and are also technically easier to construct. Therefore they are a good place to start.

Obviously if we want to unify quantum theory and geometry then a necessary first step is to cast both in the same language, which for us will be that of algebra. We have already mentioned that vector fields can be thought of classically as derivations of the algebra of functions on the manifold, and if one wants points they can be recovered as maximal ideals in the algebra, etc. This is the more of less standard idea of algebraic geometry dating from the late 19th century and early on in the 20th. It will certainly need to be modified before it works in the noncommutative case but it is a starting point. The algebraic structure on the quantum side will need more attention, however.

\section{II.A Cogravity}

We begin with some very general considerations. In fact there are fundamental reasons why one needs noncommutative geometry for any theory that pretends to be a fundamental one. Since gravity and quantum theory both work extremely well in their separate domains, this comment refers mainly to a theory that might hope to unify the two. As a matter of fact I believe that, through noncommutative geometry, this 'holy grail' of theoretical physics may now be in sight.

The first point is that we usually do not try to apply or extend our geometrical intuition to the quantum domain directly, since the mathematics for that has traditionally not been known. Thus, one usually considers quantisation as the result of a process applied to an underlying classical phase space, with all of the geometrical content there (as a Poisson manifold). But demanding any algebra such that its commutators to lowest order are some given Poisson bracket is clearly an illogical and ill-defined process. It not only does not have a unique answer but also

it depends on the coordinates chosen to map over the quantum operators. Almost always one takes the Poisson bracket in a canonical form and the quantisation is the usual CCR or canonical commutation relations algebra. Maybe this is the local picture but what of the global geometry of the classical phase space? Clearly all of these problems are putting the cart before the horse: the real world is to our best knowledge quantum so that should come first. We should build models guided by the intrinsic (noncommutative) geometry at the level of noncommutative algebras and only at the end consider classical limits and classical geometry (and Poisson brackets) as emerging from a choice, where possible, of 'classical handles' in the quantum system. 
In more physical terms, classical observables should come out of quantum theory as some kind of limit and not really be the starting point; in quantum gravity, for example, classical geometry should appear as an idealisation of the expectation value of certain operators in certain states of the system. Likewise in string theory one starts with strings moving in classical spacetime, defines Lagrangians etc. and tries to quantise. Even in more algebraic approaches, such as axiomatic quantum field theory, one still assumes an underlying classical spacetime and classical Poincaré group etc., on which the operator fields live. Yet if the real world is quantum then phase space and hence probably spacetime itself should be 'fuzzy' and only approximately modeled by classical geometrical concepts. Why then should one take classical geometrical concepts inside the functional integral except other than as an effective theory or approximate model tailored to the desired classical geometry that we hope to come out. This can be useful but it cannot possibly be the fundamental 'theory of everything' if it is built in such an illogical manner. There is simply no evidence for the assumption of nice smooth manifolds other than now-discredited classical mechanics. And in certain domains such as, but not only, in Planck scale physics or quantum gravity, it will certainly be unjustified even as an approximation.

Next let us observe that any quantum system which contains a nonAbelian global symmetry group is already crying out for noncommutative geometry. This is in addition to the more obvious position-momentum noncommutativity of quantisation. The point is that if our quantum system has a nonAbelian Lie algebra symmetry, which is usually the case when the classical system does, then from among the quantum observables we should be able to realise the generators of this Lie algebra. That is, the algebra of observables $A$ should contain the algebra generated by the Lie algebra,

$$
A \supseteq U(\mathfrak{g}) .
$$

Typically, $A$ might be the semidirect product of a smaller part with external symmetry $\mathfrak{g}$ by the action of $U(\mathfrak{g})$ (which means that in the bigger algebra the action of $\mathfrak{g}$ is implemented by the commutator). This may sound fine but if the algebra $A$ is supposed to be the quantum analogue of the 'functions on phase space', then for part of it we should regard $U(\mathfrak{g})$ 'up side down' not as an enveloping algebra but as a noncommutative space with $\mathfrak{g}$ the noncommutative coordinates. In other words, if we want to elucidate the geometrical content of the quantum algebra of observables then part of that will be to understand in what sense $U(\mathfrak{g})$ is a coordinate algebra,

$$
U(\mathfrak{g})=\mathbb{C}[?]
$$

Here ? cannot be an ordinary space because its supposed coordinate algebra $U(\mathfrak{g})$ is noncommutative. 
A concrete example is provided by Mackey quantisation of a homogeneous space. Thus, if a compact group $G$ acts on a space $M$ then it induces a metric on it such that the geodesics are basically the flows under the group action, i.e. particles move on orbits. The orbits can be quantised in one go as the algebra of observables given by the cross product

$$
\mathbb{C}[M] \rtimes U(\mathfrak{g})
$$

(in an algebraic setting, say). The natural momentum coordinates here are the Lie algebra $\mathfrak{g}$ itself and its cross relations with the position functions $\mathbb{C}[M]$ provide a curved-space analogue of the Heisenberg commutation relations. There is also a Poisson structure on $M \times \mathfrak{g}^{*}$, which is the classical phase space. $U(\mathfrak{g})$ is the Kirillov-Kostant quantisation of the $\mathfrak{g}^{*}$ part. This class of models is an example of a general principle: curvature in position space corresponds to noncommutativity of the natural momentum generators. On a general curved space it means noncommutativity of covariant derivatives.

So we need noncommutative geometry both for noncommutative phase space (due to Heisenberg type relations between position and momentum) and for noncommutative momentum space (when there is curvature). Finally, since we need a noncommutative geometric formalism anyway, we may as well allow noncommutative position space or spacetime too. Only in this way could one restore any kind of Born reciprocity or symmetry between position and momentum in the quantum theory. Or more generally, only in this way could we really imagine canonical transformations mixing position and momentum coordinates. In other words when Mackey quantisation is combined with symplectic ideas or with ideas of position-momentum symmetry one is led naturally to expect that space or spacetime too should be allowed to be noncommutative.

Let us put these arguments in a more down-to-earth manner. Thus, in conventional flat space quantum mechanics we take the $\mathbf{x}$ commuting among themselves and their momenta $\mathbf{p}$ likewise commuting among themselves. The commutation relation

$$
\left[x_{i}, p_{j}\right]=\imath \hbar \delta_{i j}
$$

is symmetric in the roles of $\mathbf{x}, \mathbf{p}$ (up to a sign). To this symmetry may be attributed such things as wave-particle duality. A wave has localised $\mathbf{p}$ and a particle has localised $\mathbf{x}$. Meanwhile, the meaning of curvature in position space is, roughly speaking, to make the natural conserved $\mathbf{p}$ coordinates noncommutative. For example, when the position space is a 3-sphere the natural momentum is $s u_{2}$. The enveloping algebra $U\left(s u_{2}\right)$ should be there in the quantum algebra of observables with relations

$$
\left[p_{i}, p_{j}\right]=\frac{\imath}{R} \epsilon_{i j k} p_{k}
$$


where $R$ is proportional to the radius of curvature of the $S^{3}$. By Born-reciprocity then there should be another possibility which is curvature in momentum space. It corresponds under Fourier theory to noncommutativity of position space. For example if the momentum space were a sphere with $m$ proportional to the radius of curvature, the natural position space coordinates would correspondingly have noncommutation relations

$$
\left[x_{i}, x_{j}\right]=\frac{\imath}{m} \epsilon_{i j k} x_{k}
$$

Mathematically speaking this is surely a symmetrical and equally interesting possibility which might have observable consequences. And if gravity is, loosely-speaking, curvature in position space or spacetime then this other effect should be called 'cogravity'. In general terms,

- For systems constrained in position space one has the usual tools of differential geometry, curvature etc., of the constrained 'surface' in position space or tools for noncommutative algebras (such as Lie algebras) in momentum space.

- For systems constrained in momentum space one has conventional tools of geometry in momentum space or, by Fourier theory, suitable tools of noncommutative geometry in position space.

Of course, we do not absolutely need noncommutative geometry to work effectively with enveloping algebras of Lie algebras. But if we wish to view (16),(17) geometrically as noncommuting coordinates then we will correspondingly need to generalise our notion of geometry. What are 'vector fields' on $U\left(s u_{2}\right)$ ? What are differential forms? And so forth. This is what we have called in 17] a 'quantum-geometry transformation' since a quantum symmetry point of view (such as the angular momentum generators in a quantum system) is turned 'up-side-down' to a geometrical one. These are nontrivial (but essentially solved) questions. Understanding them, we can proceed to construct more complex examples of noncommutative geometry which are neither $U(\mathfrak{g})$ nor $\mathbb{C}[G]$, i.e. where both quantum and geometrical effects are unified or where both gravity and cogravity are present.

Notice also that the three effects exemplified by the three equations (15)-(17) are all independent. They are controlled by three different parameters $\hbar, R, m$ (say). Of course in a full theory of quantum gravity all three effects could exist together and be unified into a single noncommutative algebra containing suitable position and momentum modes. Moreover, even if we do not know the details of the correct theory of quantum gravity, if we assume that something like Born reciprocity survives then all three effects indeed should show up in the effective theory where we consider almost-particle states with position and momenta $\mathbf{x}, \mathbf{p}$. It would require fine tuning or some special principle to eliminate any one of them. 
Finally, our choice of parameter $m$ is suggestive of mass, which may be appropriate in the case of a mass-shell in momentum space, but this is not the only source of curvature in momentum space. If this case is anything to go by, however, it does suggest the following symmetrical picture

- Curvature in position spacetime or gravity governs the background in which test particles are to move. It is related to its active gravitational mass.

- Curvature in momentum space or cogravity governs the wave equation or rules by which a test particle moves even in flat space. It is related to its passive inertial mass.

Although these remarks are all somewhat vague, we see at least that noncommutative geometrical ideas should in principle help make precise some of the deepest insights, such as Mach's principle that motivated Einstein himself 18] [1]. This approach to Planck scale physics based particularly on Fourier theory to extend the familiar $\mathbf{x}, \mathbf{p}$ reciprocity to the case of nonAbelian Lie algebras and beyond was developed by the author in the 1980s 19]. See also [17] [16].

\section{II.B Algebraic structure of quantum mechanics}

In the above discussion we have assumed that quantum systems are described by algebras generated by position and momentum. Here we will examine this a little more closely. The physical question to keep in mind is the following: what happens to the geometry of the classical system when you quantise?

To see the problem consider what you obtain when you quantise a sphere or a torus. In usual quantum mechanics one takes the Hilbert space on position space, e.g. $\mathcal{H}=L^{2}\left(S^{2}\right)$ or $\mathcal{H}=L^{2}\left(T^{2}\right)$ and as 'algebra of observables' one takes $A=B(\mathcal{H})$ the algebra of all bounded (say) operators. It is decreed that every self-adjoint such operator $a$ is an observable of the system and its expectation value in state $|\psi\rangle \in \mathcal{H}$ is

$$
\langle a\rangle_{\psi}=\langle\psi|a| \psi\rangle
$$

The problem with this is that $B(\mathcal{H})$ is the same algebra in all cases. The quantum system does know about the underlying geometry of the configuration space or of the phase space in other ways; the choice of 'polarisation' on the phase space or the choice of Hamiltonian etc. - such things are generally defined using the underlying position or phase space geometry - but the abstract algebra $B(\mathcal{H})$ doesn't know about this. All separable Hilbert spaces are isomorphic (although not in any natural way) so their algebras of operators are also all isomorphic. In other words, whereas in classical mechanics we use extensively the detailed geometrical structure, such as the choice of phase space as a symplectic manifold, all of this is not recorded very directly in the quantum system. One more or less forgets it, although it resurfaces in relation to the more 
restricted kinds of questions (labeled by classical 'handles') that one asks in practice about the quantum system. In other words,

- The true quantum algebra of observables should not be the entire algebra $B(\mathcal{H})$ but some restricted subalgebra $A \subset B(\mathcal{H})$.

The choice of this subalgebra is called the kinematic structure and it is precisely here that the (noncommutative) geometry of the classical and quantum system is encoded. This is somewhat analogous to the idea in geometry that every manifold can be visualised concretely embedded in some $\mathbb{R}^{n}$. Not knowing this and thinking that coordinates $\mathbf{x}$ were always globally defined would miss out on all physical effects that depend on topological sectors, such as the difference between spheres and tori.

Another way to put this is that by the Darboux theorem all symplectic manifolds are locally of the canonical form $\mathrm{d} x \wedge \mathrm{d} p$ for each coordinate pair. Similarly one should take (15) (which essentially generates all of $B(\mathcal{H})$, one way or another) only locally. The full geometry in the quantum system is visible only by considering more nontrivial algebras than this one to bring out the global structure. We should in fact consider all noncommutative algebras equipped with certain structures common to all quantum systems, i.e. inspired by $B(\mathcal{H})$ as some kind of local model or canonical example but not limited to it. The conditions on our algebras should also be enough to ensure that there is a Hilbert space around and that $A$ can be viewed concretely as a subalgebra of operators on it.

Such a slight generalisation of quantum mechanics which allows this kinematic structure to be exhibited exists and is quite well-known in mathematical physics circles. The required algebra is a von Neumann algebra or, for a slightly nicer theory, a $C^{*}$-algebra. This is an algebra over $\mathbb{C}$ with $\mathrm{a} *$ operation and a norm \|\| with certain completeness and other properties. The canonical example is $B(\mathcal{H})$ with the operator norm and $*$ the adjoint operation, and every other is a subalgebra.

Does this slight generalisation have observable consequences? Certainly. For example in quantum statistical mechanics one considers not only state vectors $|\psi\rangle$ but 'density matrices' or generalised states. These are convex linear combinations of the projection matrices or expectations associated to state vectors $\left|\psi_{i}\right\rangle$ with weights $s_{i} \geq 0$ and $\sum_{i} s_{i}=1$. The expectation value in such a 'mixed state' is

$$
\langle a\rangle=\sum_{i} s_{i}\left\langle\psi_{i}|a| \psi_{i}\right\rangle
$$

In general these possibly-mixed states are equivalent to simply specifying the expectation directly as a linear map \langle\rangle$: B(\mathcal{H}) \rightarrow \mathbb{C}$. This map respects the adjoint or $*$ operation on $B(\mathcal{H})$ so that $\left\langle a^{*} a\right\rangle \geq 0$ for all operators $a$ (i.e. a positive linear functional) and is also continuous with 
respect to the operator norm. Such positive linear functionals on $B(\mathcal{H})$ are precisely of the above form (19) given by a density matrix, so this is a complete characterisation of mixed states with reference only to the algebra $B(\mathcal{H})$, its $*$ operation and its norm. The expectations \langle\rangle$_{\psi}$ associated to ordinary Hilbert space states are called the 'pure states' and are recovered as the extreme points in the topological space of positive linear functionals (i.e. those which are not the convex linear combinations of any others).

Now, if the actual algebra of observables is some subalgebra $A \subset B(\mathcal{H})$ then any positive linear functional on the latter of course restricts to one on $A$, i.e. defines an 'expectation state' $A \rightarrow \mathbb{C}$ which associates numbers, the expectation values, to each observable $a \in A$. But not vice-versa, i.e. the algebra $A$ may have perfectly well-defined expectation states in this sense which are not extendible to all of $B(\mathcal{H})$ in the form (19) of a density matrix. Conversely, a pure state on $B(\mathcal{H})$ given by $|\psi\rangle \in \mathcal{H}$ might be mixed when restricted to $A$. The distinction becomes crucially important for the correct analysis of quantum thermodynamic systems for example, see [20].

The analogy with classical geometry is that not every local construction may be globally defined. If one did not understand that one would miss such important things as the BohmAharanov effect, for example. Although I am not an expert on the 'measurement problem' in the philosophy of quantum mechanics it does not surprise me that one would get into inconsistencies if one did not realise that the algebra of observables is a subalgebra of $B(\mathcal{H})$. And from our point of view it is precisely to understand and 'picture' the structure of the subalgebra for a given system that noncommutative geometry steps in. I would also like to add that the problem of measurement itself is a matter of matching the quantum system to macroscopic features such as the position of measuring devices. I would contend that to do this consistently one first has to know how to identify aspects of 'macroscopic structure' in the quantum system without already taking the classical limit. Only in this way can one meaningfully discuss concepts such as partial measurement or the arbitrariness of the division into measurer and measured. Such an identification is exactly the task of noncommutative geometry, which deals with extending our macroscopic intuitions and classical 'handles' over to the quantum system. Put another way, the correspondence principle in quantum mechanics typically involves choosing local coordinates like $\mathbf{x}, \mathbf{p}$ to map over. Its refinement to correspond more of the global geometry into the quantum world is the practical task of noncommutative geometry.

The algebraic structure of quantum theory that we have described here was used by G.W. Mackey and I. Segal in the 1960s and also became a key ingredient in axiomatic quantum field theory. From the point of view of noncommutative geometry the turning point was a theorem of Gelfand and Naimark in the 1940s that every commutative $C^{*}$-algebra corresponds to a locally compact topological space as its algebra of functions vanishing at infinity. Based on 
this, one may regarded any noncommutative $C^{*}$-algebra as 'noncommutative topological space'. Similarly a later theorem of Serre and Swann characterised a vector bundle as a finitely-generated projective module over the algebra of functions in the commutative case, so one could adopt this in the noncommutative case too. This led to the operator theory (or functional analysis) approach to noncommutative geometry developed further by A. Connes and others, as explained in Section I. Here cyclic cohomology reproduces DeRahm cohomology in the commutative case. More recently Connes has introduced an operator notion of a 'spectral triple' which apparently in the commutative case reproduces a spin manifold structure and Dirac operator; see [12].

This operator theory approach to noncommutative geometry is focused in Hilbert spaces, spectral properties of the 'Dirac operator' etc., i.e. comes out of quantum mechanical thinking in a kind of 'top-down' manner. It complements the more algebraic and 'bottom-up' approach coming out of quantum groups which is more focused in the differential geometry (e.g. $q$ deforming usual formulae in differential geometry). The two approaches certainly can and should benefit each other. For example, just because an elegant construction gives the right answer in the commutative case does not mean it is the 'right' formulation in the noncommutative case. This can only be known through experience with concrete examples that one wishes to include in the more general theory.

\section{II.C nonAbelian Fourier theory and cosmological $\gamma$-ray bursts}

We now begin to use noncommutative geometry and particularly quantum group technology to make precise some of the ideas in Section II.A about position and momentum and their correspondence through Fourier theory. We need to extend this to the nonAbelian case.

Fourier theory on $\mathbb{R}$ is of course familiar. Let us recall that it also works perfectly well for any (locally compact) Abelian group $G$. The conjugate group $\hat{G}$ is the set of characters on $G$. There is a pairing between a character $\chi$ and a group element $g$, namely to evaluate $\chi(g)$. The Fourier transform is then

$$
\mathcal{F}(f)(\chi)=\int_{G} \mathrm{~d} g f(g) \chi(g)
$$

and similarly for the inverse with the roles of $G$ and $\hat{G}$ interchanged. Its key feature is that it turns differential operators on $G$ into multiplication by functions in $\hat{G}$.

However, for nonAbelian groups one still has $\hat{G}$ as the set of irreducible representations but it does not form a group any more and it does not carry enough information to reconstruct the original group i.e. to allow Fourier transform. The latter is possible but the Fourier transformed functions are not functions on $\hat{G}$ exactly but rather they are matrix-valued 'functions' where the value at $\rho \in \hat{G}$ lies in $\operatorname{End}\left(V_{\rho}\right)$, where $V_{\rho}$ is the vector space of the representation $\rho$ and where the function is constrained to be consistent with all (iso)morphisms between different $\rho$. Put 
another way, one has to work with the entire category of representations and the morphisms between them, not only the set $\hat{G}$. This is not a bad point of view and we will return to it in Section III for quasitriangular quantum groups, but it is not a very geometrical one.

Quantum groups provide a more geometrical alternative to this which keeps the flavour of the Abelian case but at the price of generalising our notions to noncommutative geometry. Thus, for any Hopf algebra $H$, recall from (11) that if we think of it as like 'functions on a group' then the coproduct corresponds to the group product law. Hence a translation-invariant integral means in general a map $\int: H \rightarrow \mathbb{C}$ such that

$$
\left(\int \otimes \mathrm{id}\right) \Delta=1 \int .
$$

Meanwhile, the notion of plane wave or exponential should be replaced by the canonical element

$$
\exp =\sum_{a} e_{a} \otimes f^{a} \in H \otimes H^{*}
$$

where $\left\{e_{a}\right\}$ is a basis and $\left\{f^{a}\right\}$ is a dual basis. We can then define Fourier transform as

$$
\mathcal{F}: H \rightarrow H^{*}, \quad \mathcal{F}(h)=\int(\exp ) h=\left(\int \sum_{a} e_{a} h\right) f^{a} .
$$

There is a similar formula for the inverse $H^{*} \rightarrow H$. In the infinite-dimensional case on will need bases of our two mutually dual Hopf algebras and either formal powerseries or a topological completion of the tensor product (i.e. some real analysis) for this to make sense.

First of all, we check that we recover usual Fourier theory at least at some formal level. Thus, take $H=\mathbb{C}[x]$ the algebra of polynomials in one variable, as the coordinate algebra of $\mathbb{R}$. It forms a Hopf algebra with

$$
\Delta x=x \otimes 1+1 \otimes x, \quad \epsilon x=0 \quad S x=-x
$$

as an expression of the additive group structure on $\mathbb{R}$. Similarly we take $\mathbb{C}[p]$ for the coordinate algebra of another copy of $\mathbb{R}$ with generator $p$ dual to $x$ (the additive group $\mathbb{R}$ is self-dual). The two Hopf algebras $H=\mathbb{C}[x]$ and $H^{*}=\mathbb{C}[p]$ are dual to each other with $\left\langle x^{n}, p^{m}\right\rangle=(-\imath)^{n} \delta_{n, m} n$ ! (under which the coproduct of one is dual to the product of the other). The (formal) exp element and Fourier transform is therefore

$$
\exp =\sum \imath^{n} \frac{x^{n} \otimes p^{n}}{n !}=e^{\imath x \otimes p}, \quad \mathcal{F}(f)(p)=\int_{-\infty}^{\infty} \mathrm{d} x f(x) e^{\imath x \otimes p} .
$$

Apart from an implicit $\otimes$ symbol which one does not usually write, we recover usual Fourier theory. Both the notion of duality and the exponential series are being treated a bit formally but can be made precise, of course. 
On the other hand we can apply the formalism just as well to $H=\mathbb{C}[G]$ the coordinate algebra of a nonAbelian complex Lie group (for the real forms one afterwards introduces a $*-$ operation on the algebra). These can typically be understood concretely as matrix groups with $\mathbb{C}[G]$ generated by the coordinate functions $t^{i}{ }_{j}$ which assign to a group element its $i j$ matrix entry, modulo some relations (and afterwards we can take topological completions). Their coproduct according to (11) is

$$
\Delta t_{j}^{i}=t_{k}^{i} \otimes t^{k}
$$

corresponding to the matrix multiplication or group law. This quantum group is dual to the enveloping algebra $U(\mathfrak{g})$ of the associated Lie algebra $\mathfrak{g}$ with duality pairing

$$
\left\langle t^{i}{ }_{j}, \xi\right\rangle=\rho(\xi)^{i}{ }_{j}
$$

where $\rho$ is the corresponding matrix representation of the Lie algebra. The canonical element or exp is given by choosing a basis for $U(\mathfrak{g})$ and finding its dual basis. We do have integrals and hence, at least formally, a Fourier transform

$$
\mathcal{F}: \mathbb{C}[G] \rightarrow U(\mathfrak{g})
$$

and back. The action of vector fields given by elements of $\mathfrak{g}$ become multiplication in $U(\mathfrak{g})$, etc. In the reverse direction we have to take the view that $U(\mathfrak{g})$ is a noncommutative space and find an integral on it, etc. But since it is a perfectly good Hopf algebra we have no problem in doing any of this or in proving the usual properties of Fourier theory. Thus there are 'vector fields' in $U(\mathfrak{g})$ given by the action of the $t^{i}{ }_{j}$ and they Fourier transform to multiplication in $\mathbb{C}[G]$, etc.

For example, one could apply this to $H=\mathbb{C}\left[S U_{2}\right]=\mathbb{C}[a, b, c, d]$ modulo the relation $a d-b c=$ 1 (and a $*$-operation to express unitarity). It has coproduct

$$
\Delta a=a \otimes a+b \otimes c, \quad \text { etc., } \quad \Delta\left(\begin{array}{ll}
a & b \\
c & d
\end{array}\right)=\left(\begin{array}{ll}
a & b \\
c & d
\end{array}\right) \otimes\left(\begin{array}{ll}
a & b \\
c & d
\end{array}\right) .
$$

The duality pairing with $U\left(s u_{2}\right)$ in its usual antihermitian generators $\left\{e_{i}\right\}$ is

$$
\left\langle\left(\begin{array}{ll}
a & b \\
c & d
\end{array}\right), e_{i}\right\rangle=\frac{\imath}{2} \sigma_{i}
$$

defined by the Pauli matrices. Let $\left\{e_{1}^{a} e_{2}^{b} e_{3}^{c}\right\}$ be a basis of $U\left(s u_{2}\right)$ and $\left\{f^{a, b, c}\right\}$ the dual basis, then we have a Fourier transform

$$
\mathcal{F}: \mathbb{C}\left[S U_{2}\right] \rightarrow U\left(s u_{2}\right), \quad \mathcal{F}(f)=\left(\int_{S U_{2}} \mathrm{~d} u f(u) f^{a, b, c}(u)\right) e_{1}^{a} e_{2}^{b} e_{3}^{c} .
$$

Here d $u$ denotes the right-invariant Haar measure on $S U_{2}$. For a picture of $e_{i}$ as the coordinates on momentum space conjugate to position space $S U_{2}$, we have to regard $U\left(s u_{2}\right)$ as coordinates 
of a 'noncommutative space'. Or we could equally well reverse the roles of these quantum groups (i.e. focus on the inverse Fourier transform) as connecting noncommutative position space with coordinates $U\left(s u_{2}\right)$ and commutative but curved $S U_{2}$ momentum space. Note that one can certainly put in the functional analysis in both cases. For example, as $C^{*}$-algebras the role of $U(\mathfrak{g})$ is more properly played by the group $C^{*}$-algebra $C^{*}(G)$, etc.

For an even more concrete example we take the Lie algebra $\mathbb{R} \rtimes \backslash \mathbb{R}$ with generators $x, t$ and relations

$$
[x, t]=\imath \lambda x .
$$

Its enveloping algebra $U(\mathbb{R}>\mathbb{R})$ could be viewed as a noncommutative analogue of $1+1$ dimensional space-time. Its associated Lie group $\mathbb{R} \rtimes \mathbb{R}$ consists of matrices of the form

$$
\left(\begin{array}{cc}
e^{\lambda \omega} & k \\
0 & 1
\end{array}\right)
$$

and has coordinate algebra $\mathbb{C}[\mathbb{R} \rtimes \mathbb{R}]=\mathbb{C}[k, \omega]$ with coproduct

$$
\Delta e^{\lambda \omega}=e^{\lambda \omega} \otimes e^{\lambda \omega}, \quad \Delta k=k \otimes 1+e^{\lambda \omega} \otimes k
$$

Its duality pairing with $U(\mathbb{R}>\backslash \mathbb{R})$ is generated by $\langle x, k\rangle=-\imath,\langle t, \omega\rangle=-\imath$ and the resulting exp and Fourier transform are 21]

$$
\exp =e^{\imath k \omega} e^{\imath \omega t}, \quad \mathcal{F}(: f(x, t):)=\int_{-\infty}^{\infty} \int_{-\infty}^{\infty} \mathrm{d} x \mathrm{~d} t e^{\imath k x} e^{\imath \omega t} f\left(e^{\lambda \omega} x, t\right)
$$

where $: f(x, t): \in U(\mathbb{R} \rtimes \backslash \mathbb{R})$ by normal ordering $x$ to the left of $t$. This is the Fourier transform from $U(\mathbb{R} \rtimes \mathbb{R})$ as a noncommutative spacetime to $\mathbb{C}[\mathbb{R} \rtimes \mathbb{R}]$ where $\mathbb{R} \rtimes \mathbb{R}$ is the nonAbelian or 'curved' momentum space. One has to complete it in a suitable way of course.

This kind of algebra for spacetime (and its $q$-deformation, but one may set $q=1$ ) was proposed by the author in [10], where the relevant integration needed for the Fourier theory was given. In $3+1$ dimensions the same Lie algebra with $x$ replaced by a vector $\vec{x}$ was introduced in [11] as the suitable spacetime covariantly acted upon by one of the candidates for a deformed Poincaré quantum group, as we will see later. Using the methods above one can view this noncommutative spacetime $U\left(\mathbb{R}^{3} \rtimes \mathbb{R}\right)$ equivalently under Fourier theory as a theory of nonAbelian momentum group $\mathbb{R}^{3} \rtimes \mathbb{R}$. This and its physical consequences were explored recently in [9]. In particular, one is then able to justify the dispersion relation

$$
\lambda^{-2}\left(e^{\lambda \omega}+e^{-\lambda \omega}-2\right)-\vec{k}^{2} e^{-\lambda \omega}=m^{2}
$$

as a well-defined mass-shell in the classical momentum group $\mathbb{R}^{3} \succ \mathbb{R}$ and give some arguments (for the first time) that the plane waves being of the form $e^{\imath \vec{k} \cdot \vec{x}} e^{\imath \omega t}$ above would have wave velocities given by $v_{i}=\frac{\partial \omega}{\partial k_{i}}$. One then arrives at the prediction that the velocity of such 
noncommutative waves (under a lot of hypotheses concerning how they might be measured) would depend on energy as

$$
|v|=e^{-\omega \lambda}
$$

Some heuristic speculations of this type first appeared in 22] from thinking about the Casimir in the deformed Poincaré quantum group (one needs the nonAbelian Fourier theory, however, to connect this with waves in spacetime and justify $\omega$ as energy etc.)

This theoretical prediction can actually be measured for gamma ray bursts that travel cosmological distances, even if $\lambda$ is very small, of the order of the Planck scale. The known gamma-rays occur in a spread of energies from 0.1-100 Mev and are known to travel cosmological distances. Hence the accumulated difference in their arrival times

$$
\delta t=\lambda \frac{L}{c} e^{\lambda \omega} \delta \omega
$$

due to the above effect could be of the order of milliseconds, which is observable. Of course, one does not know how much of the actual spread in arrival times is due to the effect and how much is part of the initial structure. For this one needs to know the distance $L$ over many bursts and use the predicted $L$-dependence to filter out other effects and our lack of knowledge of the initial spectrum. Such better data should, however, be forthcoming in the near future. It was also conjectured in [9] that the nonAbelianness of the momentum group shows up as CPT violation and might be detected by ongoing neutral-kaon system experiments. Of course, there is nothing stopping one doing field theory in the form of Feynman rules on our classical momentum group either, except that one has to make sense of the meaning of nonAbelianness in the addition of momentum. These are the first and I believe at the moment the only concretely testable predictions for Planck scale physics coming out of noncommutative geometry, in contrast to theoretical and conceptual ideas.

\section{II.D Fourier theory and loop variables}

Fourier theory also ties up with other approaches to quantum gravity such as the loop variable one that grew out of the approach to QCD in the 1970s based on Wilson loops. And it gives another point of view on the deep connection between Chern-Simons theory, the Wess-ZuminoNovikov-Witten CFT and the knot invariants related to quantum groups. We will say more about the latter in Section III but for the moment we offer a different and more heuristic point of view based on Fourier transform. As far as I now it is due to the author in [23] [24] [25] and some aspects, such as the regularised linking number, have certainly turned up in modern developments in loop variable quantum gravity. We refer to [16] for more details.

We will discuss only the Abelian or $U(1)$ theory in any depth, leaving as open the problem of really pushing through these ideas in the nonAbelian case. The required groups are not, 
however, locally compact so there is no well-defined translation-invariant measure. However, this is a matter of regularisation and as physicists we can also apply these ideas formally by pretending that there is such a measure. With this caveat, the elements $\kappa$ of the group are disjoint unions of oriented knots (i.e. links) with a product law that consists of erasing any overlapping segments of opposite orientation. The dual group is $\mathcal{A} / \mathcal{G}$ of $U(1)$ bundles and (distributional) connections $A$ on them. Thus given any bundle and connection, the corresponding character is the holonomy

$$
\chi_{A}(\kappa)=e^{\imath \int_{\kappa} A} .
$$

The inverse Fourier transform of some well-known functions on $\mathcal{A} / \mathcal{G}$ as functions on the group of knots are,

$$
\begin{aligned}
\mathcal{F}^{-1}(\mathrm{CS})(\kappa) & =\int \mathrm{d} A \operatorname{CS}(\mathrm{A}) e^{-\imath \int_{\kappa} A}=e^{\frac{\imath}{2 \alpha} \operatorname{link}(\kappa, \kappa)} \\
\mathcal{F}^{-1}(\operatorname{Max})(\kappa) & =\int \mathrm{d} A \operatorname{Max}(\mathrm{A}) e^{-\imath \int_{\kappa} A}=e^{\frac{\imath}{2 \beta} \operatorname{ind}(\kappa, \kappa)}
\end{aligned}
$$

where

$$
\mathrm{CS}(A)=e^{\frac{\alpha \imath}{2} \int A \wedge \mathrm{d} A}, \quad \operatorname{Max}(A)=e^{\frac{\beta \imath}{2} \int * \mathrm{~d} A \wedge \mathrm{d} A}
$$

are the Chern-Simmons and Maxwell actions, link denotes linking number, ind denotes mutual inductance. The diagonal ind $(\kappa, \kappa)$ is the mutual self-inductance i.e. you can literally cut the knot, put a capacitor and measure the resonant frequency to measure it. By the way, to make sense of this one has to use a wire of a finite thickness - the self-inductance has a log divergence. This is also the log-divergence of Maxwell theory when one tries to make sense of the functional integral, i.e. renormalisation has a clear physical meaning in this context. Meanwhile, the diagonal $\operatorname{link}(\kappa, \kappa)$ is the self-linking number of a knot with itself, where $\operatorname{link}\left(\kappa, \kappa^{\prime}\right)$ between two possibly intersecting knots is defined as the limit $\epsilon \rightarrow 0$ of the regularised linking number 24.

$$
\operatorname{link}_{\epsilon}\left(\kappa, \kappa^{\prime}\right)=\int_{\| \vec{\epsilon} \mid<\epsilon} \mathrm{d}^{3} \vec{\epsilon} \operatorname{link}\left(\kappa, \kappa_{\vec{\epsilon}}^{\prime}\right)
$$

Here $\kappa_{\vec{\epsilon}}^{\prime}$ is the second knot displaced by the vector $\vec{\epsilon}$. This $\operatorname{link}\left(\kappa, \kappa^{\prime}\right)$ gives, for example, $\pm 1 / 2$ for each transversal intersection. One can also define it by the Gauss formula, which is part of the proof of the above results. Note also that this point of view is distributional because, as well as considering honest smooth connections, one considers 'connections' defined entirely by their holonomy. In particular, given a knot $\kappa$ one may define the distribution $A_{\kappa}$ by its character as

$$
e^{\imath \int_{\kappa^{\prime}} A_{\kappa}}=e^{\imath \operatorname{link}\left(\kappa, \kappa^{\prime}\right)}
$$


Such distributions are quite interesting. For example, if one formally evaluates the Maxwell action on these one has [23] [25]

$$
\operatorname{Max}\left(A_{\kappa}\right)=e^{\frac{\imath}{4 \beta} \delta^{2}(0) \int_{\kappa} \mathrm{d} t \dot{\kappa} \cdot \dot{\kappa}}
$$

the Polyakov string action. In other words, string theory can be embedded into Maxwell theory by constraining the functional integral to such 'vortex' configurations. An additional ChernSimons term becomes similarly a 'topological mass term' $\operatorname{link}(\kappa, \kappa)$ that could be added to the Polyakov action.

Now the point is that on the side of $\mathcal{A} / \mathcal{G}$ there is no problem passing to the nonAbelian case and no problem writing down Yang-Mills and Chern-Simons functionals on this space. What are their Fourier transforms? In the $S_{2}$ case the inverse Fourier transform of the ChernSimons functional should surely be some kind of Jones invariant in place of self-linking number. Actually the Jones invariant is connected only to the fundamental representation of $S_{2}$ but in a conventional setting (see later sections) there is such a knot invariant for every representation. Now, just as gauge fields are something like $U(1)$ fields 'tensored' by $U(\mathfrak{g})$, the dual should be loops 'tensored' by the dual of $U(\mathfrak{g})$. In conventional terms this would be therefore loops labelled by representations in $\hat{G}$ and the universal Jones invariant would indeed be a functional on this. There are many complications here that we have glossed over, i.e. this is a somewhat heuristic picture. First of all, as written above the group of loops is not sensitive to under or over crossings - this enters in the regularisation needed to make sense of the theory as well as in the definition of self-linking number in the answer. The same has to be done in the nonAbelian case. Secondly, there is not a simple 'tensor product' here but rather the Lie algebra and the differential structure of the gauge field are nontrivially mixed up (a gauge field is not simply a Lie-algebra valued 1-form). At least at the heuristic level, however, this is one way of thinking about knot invariants 24]. Presumably the same approaches can be applied to Fourier transform the Yang-Mills action to understand QCD and presumably also to understand loop-variable quantum gravity. Finally, our results above suggest a noncommutative geometrical formulation in which the loops would have values not in $\hat{G}$ but in $U(\mathfrak{g})$ regarded as a noncommutative space. In other words,

- NonAbelian gauge theories should be more or less equivalent under nonAbelian Fourier transform to a theory of loops with values in a manifold crossed by a noncommutative space $U(\mathfrak{g})$.

At the time of writing such models and such ideas have yet to be explored, i.e. this is a conjecture for the future.

There is also another connection with noncommutative geometry. Thus the CCR's for the 
gauge field can be equivalently formulated as

$$
\left[\int_{\kappa} A, \int_{\Sigma} E\right]=4 \pi \imath \alpha \operatorname{link}(\kappa, \partial \Sigma)
$$

which is a signed sum of the points of intersection of the loop $\kappa$ with the surface $\Sigma$. This is the point of view by which loop variables were introduced (as an approach to QCD on lattices) by Mandelstam and others. Now, just as the noncommutative torus (7) takes the Heisenberg algebra in an exponentiated form with relations

$$
v^{n} u^{m}=e^{2 \pi \imath \theta m n} u^{m} v^{n}
$$

one has for gauge fields in canonical quantisation 23] 25]

$$
v_{\kappa} u_{\kappa^{\prime}}=e^{4 \pi \imath \alpha \operatorname{link}\left(\kappa, \kappa^{\prime}\right)} u_{\kappa^{\prime}} v_{\kappa}
$$

where integers are replaced by knots or links. Here the physical picture is

$$
u_{\kappa}=e^{\imath \int_{\kappa} A}, \quad v_{\kappa}=e^{\imath \int_{\kappa} \tilde{A}}
$$

where $\tilde{A}$ is a dual connection such that $E=\mathrm{d} \tilde{A}$. So constructing the $u, v$ is equivalent to constructing some distributional operators $A, E$ with the usual CCR's. The point here is that CCR's and the noncommutative torus are but the most elementary examples of noncommutative geometry (at least at an algebraic level). As we will see in the next section, there are variations of the CCR algebra that preserve more of the geometric structure of phase space in the quantum case; one could envisage similar variants for quantisation of photons and Yang-Mills fields. This is again something for the future.

\section{II.E The Planck-scale quantum group}

We are now ready to move from simple examples like $U(\mathfrak{g})$ regarded 'up side down' as a noncommutative space to noncommutative spaces that are genuinely different from both $U(\mathfrak{g})$ and its dual $\mathbb{C}[G]$. In fact our goal is to unify these two. We do this in the category of quantum groups because quantum groups should be the simplest examples of noncommutative geometry. In this category we want to have something that really is a quantum algebra of observables of an honest quantum system and at the same time preserves something of the geometrical structure of phase space in the quantum case. In the simplest 1+1-dimensional model the phase space is $\mathbb{R}^{2}$ with its additive group structure. This in turn leads to the usual vector fields etc., on $\mathbb{R}^{2}$. We want to be able to keep all that geometry even in the quantum setting. This line of thinking led in the mid 1980 s to the Planck-scale quantum group $\mathbb{C}[x] \triangleleft_{\hbar, \mathrm{G}} \mathbb{C}[p]$ generated by $x, p$ with relations and coproduct 6 ]

$$
[x, p]=\imath \hbar\left(1-e^{-\frac{x}{\mathrm{G}}}\right), \quad \Delta x=x \otimes 1+1 \otimes x, \quad \Delta p=p \otimes e^{-\frac{x}{\mathrm{G}}}+1 \otimes p .
$$


This should be viewed as some kind of 'toy model' or effective theory of Planck-scale physics with stripped-down degrees of freedom but incorporating the more fundamental ideas in previous sections. This is how bicrossproduct quantum groups first appeared, at least in a modern context.

Notice first of all the quantum flat space $\mathrm{G} \rightarrow 0$ limit. In any situation where $x$ can effectively be treated as having values $>0$, i.e. for a certain class of quantum states where the particle is confined to this region, we clearly have flat space quantum mechanics with the Heisenberg algebra $[x, p]=\imath \hbar$ as $\mathrm{G} \rightarrow 0$. So this quantum group leads to a modification of usual quantum mechanics by this parameter $\mathrm{G}$.

To get some idea of the meaning of this deformation by $\mathbf{G}$, suppose that $p$ is the natural conserved momentum and the Hamiltonian is $h=p^{2} / 2 m$ (say). The different $[x, p]$ commutation relations then correspond to different dynamics. This is the natural point of view as a dynamical system, but if one prefers an even more conventional point of view one is free to define $\tilde{p}=$ $p\left(1-e^{-\frac{x}{G}}\right)^{-1}$, which then has the usual canonical commutation relations, with $h$ a certain Hamiltonian consisting of $\tilde{p}^{2} / 2 m$ plus derivative interaction terms. Either way, one finds

$$
\dot{p}=0, \quad \dot{x}=\frac{p}{m}\left(1-e^{-\frac{x}{G}}\right)+O(\hbar)=v_{\infty}\left(1-\frac{1}{1+\frac{x}{G}+\cdots}\right)+O(\hbar)
$$

where we identify $p / m$ to $O(\hbar)$ as the velocity $v_{\infty}<0$ at $x=\infty$. We see that as the particle approaches the origin it goes more and more slowly and in fact takes an infinite amount of time to reach the origin. Compare with the formula in standard radial in-falling coordinates

$$
\dot{x}=v_{\infty}\left(1-\frac{1}{1+\frac{1}{2} \frac{x}{G}}\right)
$$

for the distance from the event horizon of a Schwarzschild black hole with

$$
\mathrm{G}=\frac{G_{\text {Newton }} M}{c^{2}}
$$

where $M$ is the background gravitational mass and $c$ is the speed of light. Thus the heuristic meaning of $\mathrm{G}$ in our model is that it measures the background mass or radius of curvature of the classical geometry of which our Planck scale Hopf algebra is a quantisation.

Next we notice the classical limit where $\hbar \rightarrow 0$. In this case we have the commutative algebra of $x, p$ i.e. a classical space but with coproduct that of the group of matrices of the form

$$
\left(\begin{array}{cc}
e^{-\frac{x}{G}} & 0 \\
p & 1
\end{array}\right)
$$

which is therefore the classical phase space for general $G$ of the system. This is the group $\mathbb{R} \ltimes \mathbb{R}$ of lower-triangular matrices equipped with a certain Poisson bracket obtained by classicalising the above relations. Working a little harder, one finds that the quantum mechanical limit is valid (the effects of $\mathrm{G}$ do not show up within one Compton wavelength) if

$$
m M<<m_{\text {Planck }}^{2},
$$


while the curved classical limit is valid if

$$
m M>>m_{\text {Planck }}^{2}
$$

In the general case both points of view coexist in a unified structure.

We envisage that this model could appear as some effective limit of an unknown theory of quantum gravity which to lowest order would appear as spacetime and conventional mechanics on it. But actually we can make a much stronger statement, for there is a sense in which the Planck scale quantum group is not merely $a$ quantisation of a certain Poisson space but rather comes from the intrinsic structure of noncommutative algebras themselves. The idea is that even if the theory of quantum gravity is unknown, we can use the intrinsic structure of noncommutative algebras to classify a priori different possibilities. This is much as a phenomenologist might use knowledge of topology or cohomology to classify different a priori possible effective Lagrangians without knowing the full high energy theory.

Specifically, if $H_{1}, H_{2}$ are two quantum groups there is a theory of the space $\operatorname{Ext}_{0}\left(H_{1}, H_{2}\right)$ of possible extensions

$$
0 \rightarrow H_{1} \rightarrow E \rightarrow H_{2} \rightarrow 0
$$

by some Hopf algebra $E$ obeying certain conditions. We do not need to go into the mathematical details here but in general one can show that $E \cong H_{1} \bowtie H_{2}$ by a 'bicrossproduct' Hopf algebra construction. Suffice it to say that the conditions are 'self-dual' i.e. the dual of the above extension gives

$$
0 \rightarrow H_{2}^{*} \rightarrow E^{*} \rightarrow H_{1}^{*} \rightarrow 0
$$

as another extension dual to the first, in keeping with a philosophy of self-duality of the category in which we work. We also note that by Ext $_{0}$ we mean quite strong extensions. There is also a weaker notion that admits the possibilities of cocycles as well, which we are excluding, i.e. this is only the trivial sector in a certain nonAbelian cohomology. Then it was found that [6] [18]

$$
\operatorname{Ext}_{0}(\mathbb{C}[x], \mathbb{C}[p])=\mathbb{R} \hbar \oplus \mathbb{R G}
$$

a two-parameter space, and that any extension

$$
0 \rightarrow \mathbb{C}[x] \rightarrow E \rightarrow \mathbb{C}[p] \rightarrow 0
$$

of position $\mathbb{C}[x]$ by momentum $\mathbb{C}[p]$ forming a Hopf algebra is of the bicrossproduct form $E \cong \mathbb{C}[x] \triangleleft_{\hbar, \mathrm{G}} \mathbb{C}[p]$. In physical terms what we are saying is that if we are given $\mathbb{C}[x]$ the position coordinate algebra and $\mathbb{C}[p]$ defined a priori as the natural momentum coordinate algebra then all possible quantum phase spaces built from $x, p$ in a controlled way that preserves duality 
ideas (Born reciprocity) and retains the group structure of classical phase space as a quantum group are of this form labeled by two parameters $\hbar, G$. We have not put these parameters in by hand - they are simply the mathematical possibilities being thrown at us. Also, although one cannot draw too many conclusions from the analogy with a Schwarzschild black-hole (given that the toy model here is not even relativistic), the emergence of a coordinate singularity is again something that we have not put in by hand. In summary,

- The model shows how one can be forced to discover both quantum and gravitational effects from the intrinsic structure of the theory of noncommutative algebras.

- Such methods lead to tight constraints on the dynamics, with features such as coordinate singularities.

In particular, it is not possible to make a Hopf algebra for $x, p$ with the correct classical limit in this context without a coordinate singularity. In fact, solving (60) is a second order differential equation for the possible action of the momentum generators, playing the role in our toy model of something like Einstein's equation for the metric.

We can also take a 'deep quantum gravity' limit $\hbar, G \rightarrow \infty$ in the above, with $\frac{G}{\hbar}=\lambda$ held constant. In this case we obtain

$$
[x, p]=\imath \lambda x, \quad \Delta x=x \otimes 1+1 \otimes x, \quad \Delta p=p \otimes 1+1 \otimes p,
$$

which is the noncommutative spacetime $U(\mathbb{R} \rtimes \mathbb{R})$ in Section II.C, where the enveloping algebra is regarded 'up side down' as noncommutative coordinates. This puts some flesh on the idea that noncommutative spacetime or cogravity might indeed come out of quantum gravity as some kind of effective description independent of its details. The time operator would be built from the momentum modes of that deeper theory. This connects our quantum group ideas with some of the first testable predictions as explained in Section II.C.

There are some other remarkable features of the Planck-scale quantum group which could give us some qualitative insight into even more novel phenomena at the Planck scale. The most important is that it is not only of self-dual type in the sense that its dual is also a Hopf algebra extension, but it is actually isomorphic to its own dual,

$$
\left(\mathbb{C}[x] \triangleleft_{\hbar, \mathrm{G}} \mathbb{C}[p]\right)^{*} \cong \mathbb{C}[\bar{p}] \triangleright_{\frac{1}{\hbar}, \frac{\mathrm{G}}{\hbar}} \mathbb{C}[\bar{x}],
$$

where $\mathbb{C}[p]^{*}=\mathbb{C}[\bar{x}]$ and $\mathbb{C}[x]^{*}=\mathbb{C}[\bar{p}]$ in the sense of an algebraic pairing. Here $\langle p, \bar{x}\rangle=\imath$ etc., which then requires a change of the parameters as shown to make the identification precise. So there is a dual theory which has just the same form but the roles of $x, p$ interchanged and different parameter values. This means that whereas we would look for observables $a \in \mathbb{C}[x] \bowtie \mathbb{C}[p]$ as 
the algebra of observables and states $\phi \in \mathbb{C}[\bar{p}] \bowtie \mathbb{C}[\bar{x}]$ as the dual linear space, with $\phi(a)$ the expectation of $a$ in state $\phi$ (See section II.B), there is a dual interpretation whereby

$$
\text { Expectation }=\phi(a)=a(\phi)
$$

for the expectation of $\phi$ in 'state' $a$ with $\mathbb{C}[\bar{p}] \bowtie \mathbb{C}[\bar{x}]$ the algebra of observables in the dual theory. More precisely, only self-adjoint elements of the algebra are observables and only positive functionals are states, and a state $\phi$ will not be exactly self-adjoint in the dual theory etc. But the physical self-adjoint elements in the dual theory will be given by combinations of such states, and vice versa. This is what I have called observable-state duality. It was introduced in [6] in the 1980s.

Also conjectured at the time of [6] was that this duality should be related to $T$-duality in string theory. As evidence is the inversion of the constant $\hbar$. In general terms coupling inversions are indicative of such dualities. Notice also that Fourier transform implements this T-duality-like transformation as

$$
\mathcal{F}: \mathbb{C}[x] \bowtie_{\hbar, \mathrm{G}} \mathbb{C}[p] \rightarrow \mathbb{C}[\bar{p}] \bowtie_{\frac{1}{\hbar}, \frac{\mathrm{G}}{\hbar}} \mathbb{C}[\bar{x}]
$$

Explicitly, it comes out as 21

$$
\mathcal{F}(: f(x, p):)=\int_{-\infty}^{\infty} \int_{-\infty}^{\infty} \mathrm{d} x \mathrm{~d} p e^{-\imath\left(\bar{p}+\frac{\imath}{G}\right) x} e^{-\imath \bar{x}(p+p \triangleright)} f(x, p),
$$

where

$$
p \triangleright f=-\imath \hbar\left(1-e^{-\frac{x}{G}}\right) \frac{\partial}{\partial x} f
$$

and $f(x, p)$ is a classical function considered as defining an element of the Planck-scale quantum group by normal ordering $x$ to the left.

The observable-state duality here is not exactly T-duality in string theory but has some features like it. On the other hand, it is done here at the quantum level and not in terms only of Lagrangians. Rather, this approach suggests,

- A fundamental theory of physics including quantum gravity should be defined by some kind of algebraic structure possessing one or more dualities in a representation-theoretic or observable-state sense.

- Classical geometry, Lagrangians, etc. would only appear in classical limits of the algebraic structure and be related to each other under the dualit(ies).

In the above self-dual model the classical picture in the dual is the same as in the original model with a change of parameters, but for more general bicrossproducts the model and its dual 
model can be quite different in their classical mechanics. This algebraic duality point of view was introduced by the author in the 1980s in [1] [19] but we note that some similar ideas are beginning to be bandied about by string theorists a decade later under the name of 'M-theory'. This is an unknown theory but deemed but have different classical limits connected by dualities.

Finally, the Planck-scale quantum group allows us to take a first look at how classical geometry emerges or conversely how it corresponds in the quantum theory. For example, an infinitesimal coproduct defines partial differentials,

$$
\partial_{p}: f(x, p):=\frac{\mathrm{G}}{\imath \hbar}:\left(f(x, p)-f\left(x, p-\imath \frac{\hbar}{\mathrm{G}}\right)\right):, \quad \partial_{x}: f(x, p):=: \frac{\partial}{\partial x} f:-\frac{p}{\mathrm{G}} \partial_{p}: f:
$$

which shows the effects of $\hbar$ in modifying the geometry. Differentiation in the $p$ direction becomes 'lattice regularised' albeit a little strangely with an imaginary displacement. In the $\lambda$-deformed Minkowski space setting where $p=t$ it means that the Euclidean version of the theory related to the Minkowski one by a Wick-rotation is being lattice-regularised by the effects of $\hbar$.

Also note that for fixed $\hbar$ the geometrical picture blows up when $G \rightarrow 0$. I.e the usual flat space quantum mechanics CCR algebra does not admit a deformation of conventional differential calculus on $\mathbb{R}^{2}$. Similarly the associated differential forms have relations with 'functions' $f$ in the Planck-scale quantum group given by

$$
f \mathrm{~d} x=(\mathrm{d} x) f, \quad f \mathrm{~d} p=(\mathrm{d} p) f+\frac{\imath \hbar}{\mathrm{G}} \mathrm{d} f,
$$

which blow up as $G \rightarrow 0$. Thus,

- One needs a small amount of 'gravity' to be present for a geometrical picture in the quantum theory.

The higher exterior algebra looks more innocent with

$$
\mathrm{d} x \wedge \mathrm{d} x=0, \quad \mathrm{~d} x \wedge \mathrm{d} p=-\mathrm{d} p \wedge \mathrm{d} x, \quad \mathrm{~d} p \wedge \mathrm{d} p=0
$$

Starting with the differential forms and derivatives, one can proceed to gauge theory, Riemannian structures etc., in some generality. The above formulae are all from a recent work [21], where one also finds 'quantum' Poisson brackets and Hamiltonians (and in principle, Lagrangians) in the

full noncommutative theory. Such tools should help to bridge the gap between model building via classical Lagrangians, which I personally do not think can succeed at the Planck scale, and some of the more noncommutative-algebraic ideas above.

\section{II.F General construction of bicrossproducts}

The general construction for bicrossproduct quantum groups is as follows. Suppose that

$$
X=G M
$$


is a factorisation of Lie groups. Then one can show that $G$ acts on the set of $M$ and $M$ acts back on the set of $G$ such that $X$ is recovered as a double cross product $X \cong G \bowtie M$ (simultaneously by the two acting on each other). The actions are defined by considering $s u$ where $s \in M$ and $u \in G$ are in the wrong order. By unique factorisation there are elements $s \triangleright u \in G$ and $s \triangleleft u \in M$ such that $s u=(s \triangleright u)(s \triangleleft u)$. In effect, an action of $G$ on $M$ and a 'backreaction' of $M$ on $G$ are created at the same time. This was motivated in 18] from Mach's principle or the general idea that every action has 'equal and opposite' reaction.

Now, quantising the orbits of $G$ in $M$ by this action can be achieved by the cross product algebra $\mathbb{C}[M] \rtimes U(\mathfrak{g})$ (or its $C^{*}$-algebra version). The backreaction of $M$ on $G$ can similarly be used to made a semidirect coproduct structure and render us a Hopf algebra

$$
\mathbb{C}[M] \bowtie U(\mathfrak{g})
$$

The roles of the two Lie groups is symmetric and the dual is

$$
(\mathbb{C}[M] \bowtie U(\mathfrak{g}))^{*}=U(\mathfrak{m}) \bowtie \mathbb{C}[G],
$$

where $\mathfrak{m}$ is the Lie algebra of $M$. What this means that there are certain families of homogeneous spaces (the orbits of one group under the other) which come in pairs, with the algebra of observables of the quantisation of one being the algebra of expectation states of the quantisation of the other.

This is the general construction alluded to in Section I and we see that it comes out of Mach's principle in a quantum mechanical setting. There are also plenty of examples. In particular, every complexification of a semisimple Lie group factorises into its compact real form $G$ and a certain solvable group $G^{\star}$, i.e. $G_{\mathbb{C}}=G G^{\star}$, so there is at least one quantum group $\mathbb{C}\left[G^{\star}\right] \bowtie U(\mathfrak{g})$ for every Lie algebra. The dual is another quantum group $U\left(\mathfrak{g}^{\star}\right) \bowtie \mathbb{C}[G]$. Let us note that some years later, in the mid 1990s, there appeared a similar proposal for non-Abelian T-duality between a suitable sigma-model on $G$ and another on $G^{\star}$ [26] which has a similar flavour to the above except that it is at the classical level (not at the level of quantum theory as above) and applies to the evolution of strings in $G, G^{\star}$ rather than to points. The extension of sigmamodel T-duality to the full quantum theory (as well as to general factorisations $G M$ ) using such methods as above is a direction for ongoing research at the moment. We refer to [27] for a first step.

Moreover, general Hopf algebra theory shows that $\mathbb{C}[M] \bowtie U(\mathfrak{g})$ always acts covariantly on $U(\mathfrak{m})$, so the latter is always a noncommutative analogue of the space of fields on which to build some kind of 'Schroedinger' representation for the cross product. There is also a more conventional Hilbert space representation. From this point of view one could for example regard $U(\mathfrak{m})$ as space or spacetime and $\mathbb{C}[M] \bowtie U(\mathfrak{g})$ as a deformation of the enveloping algebra of a group of motions on it. 
For example, the bicrossproduct quantum group

$$
\mathbb{C}\left[\mathbb{R}^{2} \rtimes \mathbb{R}\right] \bowtie U\left(s u_{2}\right)
$$

was constructed in [28] [29] (actually as a Hopf-von Neumann algebra) and acts covariantly on $U\left(\mathbb{R}^{2} \rtimes \mathbb{R}\right)$, which is the 3 -dimensional version of the noncommutative spacetime in Section II.C. Here $M=S U_{2}^{\star}=\mathbb{R}^{2} \triangleleft \mathbb{R}$ consists of 3 -vectors $\vec{s}$ with third component $s_{3}>-1$ and with the 'curved $\mathbb{R}^{3}$ ' nonAbelian group law

$$
\vec{s} \cdot \vec{t}=\vec{s}+\left(s_{3}+1\right) \vec{t}
$$

Its Lie algebra is spanned by $t, x_{i}$ with relations $\left[x_{i}, t\right]=x_{i}$ for $i=1,2$ as discussed before. On the group $\mathbb{R}^{2} \rtimes \mathbb{R}$ there is an action of $G=S U_{2}$ by a deformed rotation. The orbits are still spheres but non-concentrically nested and accumulating at $s_{3}=-1$. This is a dynamical system and $(73)$ is its Mackey quantisation as a cross product. We have similar features as for the Planck-scale quantum group, including some kind of coordinate singularity as $s_{3}=-1$. At the same time there is a 'backreaction' of $\mathbb{R}^{2} \rtimes \backslash \mathbb{R}$ back on $S U_{2}$. Therefore the dual system, related by Fourier theory or observable-state duality, is of the same form, namely

$$
U\left(\mathbb{R}^{2} \rtimes \mathbb{R}\right) \triangleright \mathbb{C}\left[S U_{2}\right]
$$

It consists of a particle on $S U_{2}$ moving under the action of $\mathbb{R}^{2} \rtimes \mathbb{R}$. Moreover, it has a representation as motions in $U\left(s u_{2}\right)$ as some kind of noncommutative space if one wants to take that point of view.

These constructions and examples were introduced by the author in the 1980s. Since then several other examples have proven of specific interest. First of all, the $3+1$-dimensional version $U\left(\mathbb{R}^{3} \rtimes \backslash \mathbb{R}\right)$ of the above can be viewed as noncommutative Minkowski space covariant under the bicrossproduct quantum group $\mathbb{C}\left[\mathbb{R}^{3} \rtimes \mathbb{R}\right] \bowtie U\left(s_{3,1}\right)$ [11]. It was given explicitly in this work as the usual translation and Lorentz rotation and boost $p_{\mu}, M_{i}, N_{i}$ and the cross relations and coproduct

$$
\begin{gathered}
{\left[p_{0}, M_{i}\right]=0, \quad\left[p_{i}, M_{j}\right]=\epsilon_{i j k} p_{k}, \quad\left[p_{0}, N_{i}\right]=-p_{i},} \\
{\left[p_{i}, N_{j}\right]=-\delta_{i j}\left(\frac{1-e^{-2 \lambda p_{0}}}{2 \lambda}+\frac{\lambda}{2} \vec{p}^{2}\right)+\lambda p_{i} p_{j},} \\
\Delta N_{i}=N_{i} \otimes 1+e^{-\lambda p_{0}} \otimes N_{i}+\lambda \epsilon_{i j k} p_{j} \otimes M_{k}, \quad \Delta p_{i}=p_{i} \otimes 1+e^{-\lambda p_{0}} \otimes p_{i}
\end{gathered}
$$

and $p_{0}, M_{i}$ as for usual enveloping algebras. It was also shown to be (non trivially) isomorphic to a much more complicated ' $\kappa$-Poincaré algebra' which has obtained by contraction from the 
$U_{q}\left(s_{3,2}\right) q$-deformation quantum group (see Section III) but for which no covariant Minkowski space had been known (until [1] only inconsistent non-covariant actions of this quantum group on classical commutative Minkowski coordinates had been considered). This completes the mathematics behind the $\gamma$-ray bursts prediction of Section II.C.

More recently, Connes and H. Moscovici studied a quantum group of bicrossproduct form corresponding to the factorisation

$$
\operatorname{Diff}(\mathbb{R})=\operatorname{Diff}_{0}(\mathbb{R}) \cdot \mathbb{R} \rtimes \mathbb{R}
$$

of diffeomorphisms into affine transformations $\mathbb{R} \rtimes \backslash \mathbb{R}$ and diffeomorphisms fixing the origin and the tangent space at the origin. Using the algebraic bicrossproduct construction above we have a bicrossproduct quantum group

$$
\mathbb{C}[\mathbb{R} \rtimes \mathbb{R}] \bowtie U\left(\operatorname{diff}_{0}(\mathbb{R})\right)
$$

as well as a covariant action of it on the noncommutative spacetime $U(\mathbb{R} \rtimes \mathbb{R})$. This covariant action as well as the entirely algebraic version of the construction go a little beyond [30]. In fact, what is more relevant there is actually the dual model

$$
U(\mathbb{R} \rtimes \mathbb{R}) \bowtie \mathbb{C}\left[\operatorname{Diff}_{0}\right]
$$

where the coordinate algebra of the diffeomorphisms was described as a certain polynomial algebra in a countable number of generators $\delta_{n}$. Next, just as the basis $u^{n}, v^{n}$ in Section II.D for the noncommutative torus could be taken as a model for physics with integer $n$ replaced by knots, Connes and D. Kreimer 31] proposed a variant of the above with $\delta_{n}$ replaced by a labelling by rooted trees as a book-keeping device for overlapping divergences in the renormalisation a general quantum field theory. This is certainly an interesting direction for current research.

\section{Quantum groups and $q$-deformation}

In this section we want to turn to the other and in many ways more famous class of quantum groups, the deformed enveloping algebras $U_{q}(\mathfrak{g})$ and their associated coordinate algebras $\mathbb{C}_{q}[G]$. The simplest and most well-known example of this type is the quantum group $U_{q}\left(s u_{2}\right)$ with generators $H, X_{ \pm}$and relations and coproduct

$$
\begin{gathered}
{\left[H, X_{ \pm}\right]= \pm 2 X_{ \pm}, \quad\left[X_{+}, X_{-}\right]=\frac{q^{H}-q^{-H}}{q-q^{-1}}} \\
\Delta X_{ \pm}=X_{ \pm} \otimes q^{\frac{H}{2}}+q^{\frac{-H}{2}} \otimes X_{ \pm}, \quad \Delta H=H \otimes 1+1 \otimes H .
\end{gathered}
$$

The coproduct $\Delta$ here is a deformation of the usual additive one, which is recovered as $q \rightarrow 1$. The deformation modifies how an action of $X_{ \pm}$extends to tensor products. 
Likewise, the coordinate algebras $\mathbb{C}_{q}[G]$ are deformations of the classical coordinate algebras $\mathbb{C}[G]$. As such one could say mathematically that they are 'quantisations' of the latter. This is not quantum physics, however, because any noncommutative algebra which is a nice deformation (a flat one) of a commutative coordinate algebra of classical space implies (by looking at the deformation to lowest order) a Poisson structure in the classical space of which the noncommutative algebra could be viewed as 'quantisation'; this is tautological and it does not necessarily come from these quantum groups arising as the algebra of observables of any natural quantum system (in contrast to the bicrossproduct quantum groups). Rather, the $U_{q}(\mathfrak{g})$ have their structure dictated by their roles as generalised symmetries. Their key property is that their representations form a braided category and that anything on which they act acquires braid statistics. Afterwards one may indeed semiclassicalise and obtain new ideas for Poisson geometry.

This is more or less but not quite what happened. On the one hand quantum groups arose as symmetries in exactly solvable lattice models and provided a new interpretation of the "corner transfer matrix method' that had been developed by Baxter in the 1970s. On the other hand there had independently been developing, particularly in the then-Soviet Union, a theory of classical integrable systems and 'classical inverse scattering' for soliton equations; see [32] for a review. Initially this was done through a notion of Lax pairs and zero curvature equations but at the same time it turned out that many such systems could be formulated in terms of a 'classical Yang-Baxter equation' that one obtains by semiclassicalising the methods for exactly solvable lattice models. So quantum groups indeed turned out to form a bridge between these schools, and it could be said that all three influenced each other in their development.

Nevertheless the role of inducing braid statistics would appear to be deeper than the 'quantisation' point of view here and therefore I will concentrate on this. It leads ultimately to a whole new kind of 'braided geometry' somewhat different from the noncommutative geometry in the preceding section. As explained in the introduction, the true meaning of the parameter $q$ in this context is that it controls the braiding matrix that generalises the minus sign in bose-fermi statistics to 'braid statistics'. This is the minus sign that is the origin of the Pauli exclusion principle that two electrons cannot be in exactly the same state. Note that the latter has a vague similarity with the Heisenberg uncertainty principle itself; in fact the idea of noncommutativity as in quantisation and the idea of braid statistics are intimately related both mathematically and physically. 


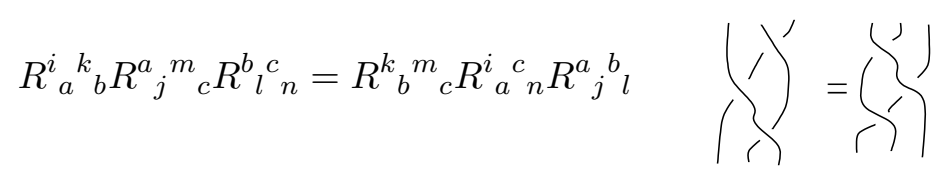

Figure 3: Braid relation and corresponding Yang-Baxter equation for braiding $\Psi$ as a matrix $R$.

\section{III.A Knot invariants}

Thus, if $V, W$ are representations of $U_{q}(\mathfrak{g})$ then $V \otimes W$ is (as for any quantum group) also a representation. The action is

$$
h \triangleright(v \otimes w)=(\Delta h) \cdot(v \otimes w)
$$

for all $h \in U_{q}(\mathfrak{g})$, where we use the coproduct (for example the linear form of the coproduct of $H$ means that it acts additively). The special feature of quantum groups like $U_{q}(\mathfrak{g})$ is that there is an element $\mathcal{R} \in U_{q}(\mathfrak{g}) \bar{\otimes} U_{q}(\mathfrak{g})$ (the 'universal R-matrix or quasitriangular structure') which ensures an isomorphism of representations

$$
\Psi_{V, W}: V \otimes W \rightarrow W \otimes V, \quad \Psi_{V, W}(v \otimes w)=P \circ \mathcal{R} .(v \otimes w)
$$

where $P$ is the usual permutation or flip map. This braiding $\Psi$ behaves much like the usual transposition or flip map for vector spaces but does not square to one. To reflect this one writes $\Psi=\lambda^{\prime}, \Psi^{-1}=\aleph$. It has properties consistent with the braid relations, i.e. when two braids coincide the compositions of $\Psi, \Psi^{-1}$ that they represent also coincide. The fundamental braid relation of the braid group corresponds to the Yang-Baxter or braid relation for the braiding $\Psi$. This is shown in Figure 3 in terms of the matrix $R$ associated to $\Psi$ on choosing bases for the vector spaces. This 'R-matrix' is just the image of $\mathcal{R}$ in the relevant representations.

From this one can see how such quantum groups lead to knot invariants 33]. Thus, consider the knot as describing the trajectories of particles $V$ and antiparticles $V^{*}$ with time flowing down the page as in Figure 4(b). Note that rather than thinking of a single kind of particle moving along the knot (as in Figure 4(a)) we instead regard the upward arcs as antiparticles flowing down the page. When one particle passes over or under another, we apply some kind of operation $R$ according to the flavour of the crossing. In this way we 'scan' the knot from top to bottom, creating particles as needed, interacting them at the crossings and finally fusing particles and antiparticles as needed. The total process is computable and, very roughly speaking, is the knot invariant as a function of any parameter $q$ on which the matrices $R$ might depend.

Note that this process exists not in three dimensions (where our original knot lived) but for particles and antiparticles moving in one space and one time dimension, according to how the knot looks on the page. We need to know that if we drew the knot from a different angle and 
(a)

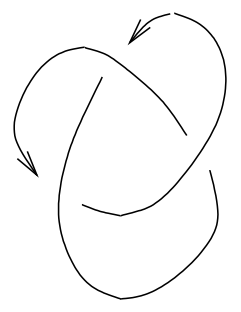

(b)

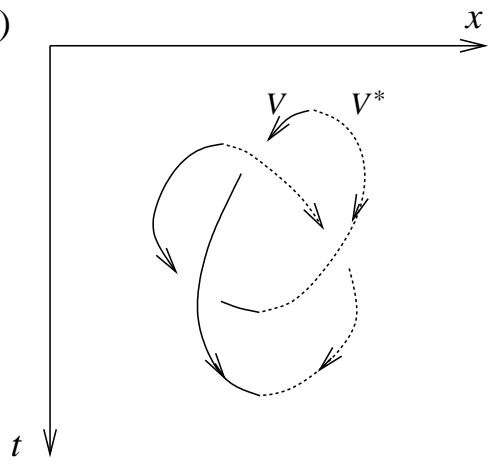

Figure 4: Trefoil knot (a) and construction (b) of its invariant

did our process from that point of view, we should get the same answer. We also need to know that if we distort the knot without cutting it then we get the same answer, all of which depends on choosing $R$ carefully. The latter part of the problem can be reduced mainly to the braid relation in Figure 3. These braids are topologically the same so replacing one by the other in a complicated knot would not change it. Therefore we require that the corresponding operations $R$ should give the same total process on three particles, which is the Yang-Baxter equation. We also require similar relations where some strands are antiparticles. The remainder of the problem can be focused mainly on the observation that a harmless twist in the knot can appear untwisted when viewed from a different angle, so that the number of crossings themselves can change. In typical examples, the matrices $R$, while not invariant under such harmless twists, usually change in a simple way that can be compensated for by hand. Actually, what one obtains in this way is not exactly a knot invariant but an invariant of ribbons or framed knots. Apart from these subtleties, these are the main constraints that the matrices $R$ have to satisfy.

Also, in particle physics one understands particles as labelled by representations $V$ of a Lie algebra $\mathfrak{g}$, and their conjugates by the dual representation $V^{*}$. When particles are interchanged one usually has either an exchange factor $R=-1$ (for Fermionic particles like the electron) or the trivial exchange $R=1$ (for Bosonic particles like the photon). Neither of these choices give interesting knot invariants, but when we look instead at representations of the quantum groups $U_{q}(\mathfrak{g})$ we find a much more nontrivial matrix $R$ (depending on $q$ ) whenever two representations of the quantum group are exchanged. We can then proceed as with the heuristic particle picture above but with $V$ a representation of the quantum group $U_{q}(\mathfrak{g})$ and $V^{*}$ its dual representation. They are created together as the canonical element of $V \otimes V^{*}$ (or a certain other element of $\left.V^{*} \otimes V\right)$ and are fused by the evaluation map $V^{*} \otimes V \rightarrow \mathbb{C}$ (or a certain other map $V \otimes V^{*} \rightarrow \mathbb{C}$ ). The result is a function of $q$ and this, more precisely, is the construction of the knot invariants from quantum groups. 
For standard $U_{q}(\mathfrak{g})$ and generic $q$ the construction of representations is not hard, all the standard ones of $\mathfrak{g}$ just $q$-deform. For example, the spin $\frac{1}{2}$ representation of $s u_{2}$ deforms to a 2-dimensional representation of $U_{q}\left(s u_{2}\right)$. The associated knot invariant is the Jones polynomial knot invariant 34. Jones himself came to this more from the solvable lattice point of view (see below), with the quantum groups point of view coming later.

There is a lot more to the deep mathematical structure of the $U_{q}(\mathfrak{g})$ that is not that much applied so far in physics. In particular, there is an important subalgebra $U_{q}\left(n_{-}\right) \subseteq U_{q}(\mathfrak{g})$ which can be used to generate representations of the whole quantum group from a highest-weight 'vacuum' vector. G. Lusztig in the early 1990s introduced a nice description of this subalgebra in terms of certain (shifted) 'perverse sheaves', using concepts from algebraic geometry [35]. Without going into details, he obtained in this way a basis of the subalgebra with many remarkable integrality and positivity properties, called the Kashiwara-Lusztig canonical basis (a similar 'global crystal basis' was found by Kashiwara at about the same time). The most remarkable property of this basis is that it induces a basis of every highest weight representation that $U_{q}\left(n_{-}\right)$generates. This might seem esoteric but all these results continue to hold even when $q=1$, and as such they provided unsuspected and revolutionary results in the representation theory of ordinary Lie algebras $\mathfrak{g}$ themselves. The algebras of negative roots $U_{q}\left(n_{-}\right)$themselves are not actually quantum groups but braided versions or 'braided groups' as introduced by the author (see a later subsection). Using some of the theorems for these objects, one has further results such as an inductive construction of the $U_{q}(\mathfrak{g})$ as a decomposition into a series of quantum-braided planes [36] [37]. Again, the result is useful even for $U(\mathfrak{g})$ and is relevant to their noncommutativegeometric picture as in Section II.

\section{III.B Exactly solvable lattice models}

Here we give the briefest of expositions of how, at least conceptually, quantum groups and braiding matrices $R$ came out of solid state physics in the early 1980s. This is surely one of the great triumphs of the interaction between physics and pure mathematics.

We recall that in statistical mechanics one has a large collection of distinct states of the system and studies its bulk properties through the partition function, a certain weighted sum over the states. For example, consider the model of a crystal in Figure 5, where a state is an assignment of bonds throughout the lattice. We write the Boltzmann weight at each vertex as the entry of a matrix $R$ according to the value of the bonds around the vertex. The partition function is

$$
Z(\lambda)=\sum_{\text {states }} \prod_{\text {vertices }} R_{j}^{i k}{ }_{l}^{k}(\lambda)
$$

where $i j k l$ are the values of the bonds in the given state surrounding the given vertex. We 


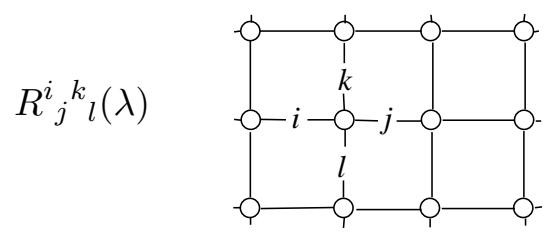

Figure 5: Solvable lattice model in statistical mechanics has weight $R$ at each vertex

suppose the weight depends on a parameter $\lambda$. Working in a different (but broadly equivalent) setting, R. Baxter in the early 1970s described conditions on $R$ which allowed for the partition function to be computed explicitly using a 'corner transfer matrix method' 38]. The resulting functions often had beautiful connections with number theory and the theory of modular forms (not connected with A. Wiles' recent proof of Fermat's theorem but in the same general ballpark). The required conditions were that $R$ depends on a parameter $\lambda$ and obeys a parameterised version of the Yang-Baxter equation in Figure 3. So the key idea behind the knot invariants also makes these models solvable. Later on, I. Sklyanin, L.D. Faddeev and others recast the corner transfer matrix method more algebraically in terms of an abstract algebra with generators $t^{i}{ }_{j}(\lambda)$ and relations

$$
R_{a b}^{i k}(\lambda+\mu) t^{a}{ }_{j}(\lambda) t_{l}^{b}(\mu)=t^{k}{ }_{b}(\mu) t_{a}^{i}(\lambda) R^{a}{ }_{j}{ }_{l}(\lambda+\mu) .
$$

Using the Yang-Baxter equations many times it is easy to see that the product of the weights along an entire row of the lattice and with $i, j$ on the ends of the row,

$$
\left(t^{i}{ }_{j}\right)_{L}^{K}=R_{a_{1}{ }^{k_{1}} l_{1}}^{k_{1}} R_{a_{2}}^{a_{1} k_{2} l_{2}} \cdots R^{a_{N-1}{ }_{j} k_{N}} l_{N}
$$

is a matrix representation of the above algebra. Here there are $N$ columns, say, and $K=$ $\left(k_{1}, \cdots, k_{N}\right)$ etc. is a multiindex. If we are interested in periodic boundary conditions then we should consider the trace $T(\lambda)=t^{i}{ }_{i}(\lambda)$. On the other hand for any operators obeying the relations (87) it is easy to see that

$$
[T(\lambda), T(\mu)]=0
$$

for all $\lambda, \mu$ i.e. the single row transfer matrices $T(\lambda)$ form an infinite number of mutually commuting operators. This is the origin of the exact solvability of the models. The partition function itself is the trace over the multiindex of the $T(\lambda)$. Another variant of this method based on open or twisted boundary conditions involved instead a 'reflection' form of (87) where two $R$ 's appear on both sides.

One does not actually need the abstract algebra here - it is just a convenient way to encode certain computations involving repeated use of the Yang-Baxter equations. On the other hand, 
there is a kind of formal coproduct structure

$$
\Delta t^{i}{ }_{j}(\lambda)=t^{i}{ }_{a}(\lambda) \otimes t^{a}{ }_{j}(\lambda)
$$

(forming a quantum group without antipode) which is the underlying reason that (88) is a representation, namely it is the repeated tensor product of the 1-column representation $\left(t^{i}{ }_{j}\right)^{k}=$ $R_{j}^{i}{ }_{j}{ }_{l}$ provided by $R$ itself (which is easier to see - it is just the Yang-Baxter equation). This observation is not true for the other 'reflection' variant (which tends to be more like a braided group as we will see in a later section). More importantly, the focus on an abstract algebra (87) suggested the possibility to realise such algebras in terms of other simpler algebras by means of various ansatze. One of the models was the so-called XXZ model consisting of nearest-neighbour spin interactions and a uniform magnetic field (controlled by a parameter $q$ ) running through the lattice, and this turned out to be realised in terms of the quantum group $U_{q}\left(s u_{2}\right)$. So this quantum group is in the background of this model playing the role of inducing the required $R(\lambda)$-matrices. One can also focus on the finite-dimensional version of the above by looking at $R(\infty)$ or $R(0)$ etc. which takes us to the simpler $R$-matrices used above for the construction of knots.

We should also consider the continuum limits of such models as the lattice spacing tends to zero. In many cases one obtains a conformally-invariant quantum field theory. Such 'conformal field theories' turned out to have their own rich algebraic structure of vertex algebras and were connected with modular forms, the 'monster group' and other topics. One of them (the Wess-Zumino-Novikov-Witten model) underlies the quantum group knot invariants above. And the classical mechanical systems underlying the continuum limits of the exactly solvable lattice models turned out to be a certain non-linear but completely integrable partial differential equations with 'soliton' solutions. So one can trace a certain continuity of ideas through several key developments in mathematical physics.

\section{III.C $q$-coordinate algebras and Poisson-Lie groups}

If one did want to take a quantisation point of view then one should consider not so much $U_{q}(\mathfrak{g})$ as we have done above but their coordinate algebra $\mathbb{C}_{q}[G]$. These are also suggested by the lattice model picture.

We consider first of all the quantum plane $\mathbb{C}_{q}[x, y]$. This is the algebra generated by variables $x, y$ but with the relations

$$
y x=q x y
$$

instead of commutativity. Here $q$ is a non-zero numerical parameter. When $q=1$ we can consider $x, y$ as the coordinates on an actual plane as we did above, but when $q \neq 1$ the algebra 
is noncommutative and hence there is no usual space underlying it. We also have higherdimensional quantum spaces of many kinds depending on the relations and parameters. In particular, the quantum group $\mathbb{C}_{q}\left[S U_{2}\right]$ has generators $a, b, c, d$ with the six relations

$$
\begin{gathered}
b a=q a b, \quad d c=q c d, \quad c a=q a c, \quad d b=q b d \\
b c=c b, \quad a d-d a=\left(q^{-1}-q\right) b c
\end{gathered}
$$

which describe a 4-dimensional $q$-space (they become the relations of commutativity when $q=1$ ), and the additional relation

$$
a d-q^{-1} b c=1
$$

which sets the ' $q$-determinant' to 1 . There is also a $*$ operation to express unitarity. There is nothing much that need concern us about the exact form of the above relations; the main thing is that as $q=1$ they recover the commutativity and determinant relations that we expect for the coordinates on the classical group $S U_{2}$ of $2 \times 2$ matrices of determinant 1 . Their exact form is, however, fine-tuned in such a way that various properties of $2 \times 2$ matrices and their action on vectors go through even when $q \neq 1$. Thus, if $x, y$ generate a quantum plane then

$$
x^{\prime}=a x+b y, \quad y^{\prime}=c x+d y
$$

obey the relations $y^{\prime} x^{\prime}=q x^{\prime} y^{\prime}$ of the quantum plane as well. In mathematical terms this 'quantum transformation' is an algebra map $\Delta_{L}: \mathbb{C}_{q}[x, y] \rightarrow \mathbb{C}_{q}\left[S U_{2}\right] \otimes \mathbb{C}_{q}[x, y]$ called a 'coaction'. Note that the arrow goes in the reverse direction to what one might have expected if one thought that an actual matrix was being combined with a vector to give another vector.

To complete the picture here, we need to check that the group structure itself is expressed in our algebraic language. In the above example, the ability to multiply two matrices to get a third matrix corresponds to the assertion that if $a^{\prime}, b^{\prime}, c^{\prime}, d^{\prime}$ are a second mutually commuting copy of $\mathbb{C}_{q}\left[S U_{2}\right]$ then

$$
\left(\begin{array}{ll}
a^{\prime \prime} & b^{\prime \prime} \\
c^{\prime \prime} & d^{\prime \prime}
\end{array}\right)=\left(\begin{array}{ll}
a & b \\
c & d
\end{array}\right)\left(\begin{array}{ll}
a^{\prime} & b^{\prime} \\
c^{\prime} & d^{\prime}
\end{array}\right)
$$

obeys the same relations. In mathematical terms the group law is expressed as an algebra map $\Delta: \mathbb{C}_{q}\left[S U_{2}\right] \rightarrow \mathbb{C}_{q}\left[S U_{2}\right] \otimes \mathbb{C}_{q}\left[S U_{2}\right]$ which is the coproduct of the quantum group. It has the same matrix form $\Delta a=a \otimes a+b \otimes c$ etc., as for $\mathbb{C}\left[S U_{2}\right]$ in Section II.C. These constructions are algebraic but one can cast them into an operator algebra setting as in Section II 39 .

Next we note that in the above example and many like it the $q$-commutativity relations can be cast as

$$
R_{a b}^{i{ }_{b} t^{a}{ }_{j} t^{b}{ }_{l}=t^{k}{ }_{b} t_{a}^{i} R^{a}{ }_{j l}^{b}}
$$


for suitable $R$ obeying the Yang-Baxter equations. This is a 'constant' version [40] of (87) focussed on $\lambda=\infty$ or $\lambda=0$ etc. (one has to make some other equivalences to get to the actual form of $R$ from the more physical $R(\lambda)$ and one should also note that in Section III.B the generators are more related to enveloping algebras than to coordinate algebras). Initially it was often mistakenly written that the Yang-Baxter relation is what makes these algebras $A(R)$ into quantum groups, which is not at all true. In fact for any tensor $c_{i_{1} \cdots i_{n}}{ }^{{ }_{1} \cdots j_{m}}$ there is a quantum group (without antipode) $M(c)$ with a matrix $t^{i}{ }_{j}$ of generators and the relations

$$
c_{a_{1} \cdots a_{n}}{ }^{j_{1} \cdots j_{m}} t^{a_{1}}{ }_{i_{1}} \cdots t^{a_{n}}{ }_{i_{n}}=t_{b_{1}}^{j_{1}} \cdots t^{j_{m}} b_{m} c_{i_{1} \cdots i_{n}}{ }^{b_{1} \cdots b_{m}} .
$$

For example, $c_{j k}^{i}$ could be the structure constants of an associative algebra and then $M(c)$ is its universal comeasuring (or 'automorphism') quantum group 41]. Rather, the meaning of $R$ obeying the Yang-Baxter equations is that this ensures that the comodules of $A(R)$ form a braided category, i.e. that quantum groups such as $\mathbb{C}_{q}\left[S U_{2}\right]$ are quasitriangular in a comodule sense.

Probably the most immediate significance of these quantum group coordinate algebras $\mathbb{C}_{q}[G]$ is that one can consider them formally as 'quantisations' of interesting Poisson brackets on the classical group $G$ (even though they are not really the algebra of observables of a true quantum system and $q$ need not be related to Planck's constant). The Poisson brackets so obtained by semiclassicalisation are always degenerate at the group identity, so this kind of Poisson bracket was missed by those focusing on symplectic manifolds only. Instead they form a Poisson-Lie group in the sense that the group product $G \times G \rightarrow G$ respects the Poisson structure (taking the direct product Poisson structure on $G \times G)$. Among the matrix $t^{i}{ }_{j}$ of coordinates in $\mathbb{C}[G]$ the Poisson bracket has the form

$$
\left\{t^{i}{ }_{j}, t^{k}{ }_{l}\right\}=t^{i}{ }_{a} t^{k}{ }_{b} r^{a}{ }_{j}{ }_{l}-r^{i}{ }_{a}{ }_{b} t^{a}{ }_{j} t^{b}{ }_{l}
$$

where $r$ is the lowest order deviation from the identity matrix of $R$. Clearly $r$ obeys an infinitesimal version of the Yang-Baxter equation, called the 'classical Yang-Baxter equation'. The abstract picture here is perhaps more easily seen in a dual form as the lowest order part in the deformation from $U(\mathfrak{g})$ to $U_{q}(\mathfrak{g})$. Thus the deformation of the coproduct to lowest order is a 'Lie cobracket' $\delta: \mathfrak{g} \rightarrow \mathfrak{g} \otimes \mathfrak{g}$ forming a Lie coalgebra (so that $\mathfrak{g}^{*}$ is a Lie algebra) and respecting the Lie bracket of $\mathfrak{g}$ in a suitable sense. This is the infinitesimal analogue of a quantum group and is called a Lie bialgebra 42]. Such a $\delta$ typically extends to all of $G$ as a bivector field which defines the Poisson bracket. In the quasitriangular case as above it has the special form $\delta \xi=\operatorname{ad}_{\xi}(r)$ where $r \in \mathfrak{g} \otimes \mathfrak{g}$ obeys the abstract classical Yang-Baxter equation

$$
\left[r_{12}, r_{13}\right]+\left[r_{12}, r_{23}\right]+\left[r_{13}, r_{23}\right]=0 \text {. }
$$


The numerical suffices here denote in which factor of $\mathfrak{g} \otimes \mathfrak{g} \otimes \mathfrak{g}$ one should view the two legs of $r$. The latter is the leading deviation from 1 of the quasitriangular structure of $U_{q}(\mathfrak{g})$ mentioned in Section III.A.

These ideas have allowed mathematicians to go back and understand many constructions in conventional Lie theory in a more elegant and natural manner, as well as to obtain entirely new results. They also allow one to present a cleaner treatment (at the level of finite-dimensional Lie algebras) of the integrability of the classical mechanical systems underlying the solvable lattice models of Section III.B. At least at the simplified level an outline is as follows. Suppose that a Lie group $X$ factorises into $G M$ and its Lie algebra $\mathfrak{x}$ has a nondegenerate ad-invariant bilinear form $K$. Using the latter one can view the difference of the projection operators $\pi_{ \pm}$ on $\mathfrak{x}$ corresponding to $\mathfrak{x}=\mathfrak{g} \oplus \mathfrak{m}$ as defining a solution $r \in \mathfrak{x} \otimes \mathfrak{x}$. This equips $X$ with a certain Poisson bracket. Now given a choice of Ad-invariant Hamiltonian function $h$ on $X$ one may analyse the induced Hamilton-Jacobi equations of motion in terms of $G, M$ and find that they are completely solvable. In fact the evolution of $x \in X$ is given by the Adler-Kostant-Symes theorem

$$
x_{t}=s_{t} x_{0} s_{t}^{-1}=u_{t}^{-1} x_{0} u_{t},
$$

where $u_{t}, s_{t}$ are paths in $G, M$ determined by the factorisation

$$
e^{-t K^{-1} \circ L_{x_{0}}^{*}(\mathrm{~d} h)}=u_{t} s_{t}
$$

of an exponential flow in $X$. Here $L_{x *}$ denotes the differential of left multiplication in $X$. For the actual nonlinear integrable systems of interest with solitons etc., one should work with a parameterised version of similar constructions. In this case the factorisation is typically that of loop groups into loops in a target group that are analytic outside and inside the unit disc in the parameter space, which is the classic Riemann-Hilbert factorisation problem. We refer to [43] for a fuller treatment and the relation with Lax pairs and other topics. This makes a little more precise the remarks at the end of Section III.B.

\section{III.D Braided geometry and $q$-spacetime}

After the great success of the quantum groups $U_{q}(\mathfrak{g})$ there was a period in the 1990 s when physicists enthusiastically went about $q$-deforming everything they could think of where Lie groups has been involved. One has $q$-oscillators [4] 45], $q$-Brownian motion, etc. etc. It is not clear what it all adds up to in the longer term but there clearly does appear to be a natural (if not exactly unique) $q$-deformation of almost everything.

And according to what we have said in the introduction, the systematic way to go about doing this was braided geometry. This is because the braiding is the key property of the quantum 
groups $U_{q}(\mathfrak{g})$ and other 'quasitriangular Hopf algebras' of similar type. It meant in particular that any algebra on which the quantum group acts covariantly becomes braided, which was therefore indicative of a whole braided approach to noncommutative geometry via algebras or 'braided' spaces on which quantum groups $U_{q}(\mathfrak{g})$ act as generalised symmetries. Note that we are not so much interested from this point of view in the noncommutative geometry of the quantum groups $U_{q}(\mathfrak{g})$ themselves, although one can study this as a source of mathematical examples. This systematic braided approach was introduced by the author at the end of the 1980s; see [2] [3] for reviews.

To get an immediate flavour for what these ideas mean in practice, consider the following elementary computation. For a polynomial function $f$ in one variable, define differentiation by

$$
f^{\prime}(y)=\left(x^{-1}(f(x+y)-f(y))\right)_{x=0} .
$$

If $x y=y x$ is assumed in making the calculation, one obtains the usual Newtonian differentiation. But if we suppose $y x=q x y$ in computing the right hand side, for some parameter $q$, we obtain

$$
f^{\prime}(x)=\frac{f(x)-f(q x)}{(1-q) x} .
$$

This is the celebrated ' $q$-deformed derivative', so called because it tends to the usual derivative as $q \rightarrow 1$. Although known to mathematicians in a different context in 1908 [46], such $q$-derivatives have their natural place in the geometry of quantum groups. We also see by this example that noncommutativity leads to a kind of 'finite-difference' or discretisation, which is therefore a general feature of the differential geometry of the quantum world and also in keeping with applications to lattice models. This point of view also leads to the correct properties of integration. Namely there is a relevant indefinite integration to go with $\partial_{q}$ characterised by 47]

$$
\int_{0}^{x+y} f=\int_{0}^{x} f(()+y)+\int_{0}^{y} f
$$

provided $y x=q x y$, etc. In the limit this gives the infinite Jackson integral previously known in this context. One also has braided exponentials, braided Fourier theory etc., for these braided variables. What is going on here is that one is working not with the usual line with bosonic coordinate $x$ but with the braided line. This is the braided group $B=\mathbb{C}[x]$, which is the usual polynomial algebra but with braid statistics, such that two copies of the braided line have the relations $y x=q x y$. The addition $x+y$ refers to an additive braided group structure or coproduct.

This is a powerful point of view and more systematic than simply sprinkling in $q$ by trial and error. For example, we have $B=\mathbb{C}_{q}[x, y]$ the quantum-braided plane generated by $x, y$ with the relations $y x=q x y$, where two independent copies have the braid statistics

$$
x^{\prime} x=q^{2} x x^{\prime}, \quad x^{\prime} y=q y x^{\prime}, \quad y^{\prime} y=q^{2} y y^{\prime}, \quad y^{\prime} x=q x y^{\prime}+\left(q^{2}-1\right) y x^{\prime} .
$$


Here $x^{\prime}, y^{\prime}$ are the generators of the second copy of the plane. There is again an additive braided coproduct in the sense that $x+x^{\prime}, y+y^{\prime}$ is another copy of the quantum-braided plane, i.e. one can check that

$$
\left(y+y^{\prime}\right)\left(x+x^{\prime}\right)=q\left(x+x^{\prime}\right)\left(y+y^{\prime}\right)
$$

And by similar definitions as above, one has braided partial derivatives

$$
\partial_{q, x} f(x, y)=\frac{f(x, y)-f(q x, y)}{(1-q) x}, \quad \partial_{q, y} f(x, y)=\frac{f(q x, y)-f(q x, q y)}{(1-q) y}
$$

for expressions normal ordered to $x$ on the left, etc. Note in the second expression an extra $q$ as $\partial_{q, y}$ moves past the $x$ to act on $y$.

Thus you can add points in the quantum-braided plane, and then (by an infinitesimal addition) define partial derivatives etc. One then has multivariable $q$-exponentials and so on. This is a problem (multivariable $q$-analysis) which had been open since 1908 and was systematically solved in the early 1990s by the braided approach [48]. We note in passing that $y x=q x y$ is sometimes called the 'Manin plane'. Manin considered only the algebra and a quantum group action on it, without the braided addition law and the braided approach.

Finally, there is a more formal way by which all such constructions are done systematically, which we now explain. It amounts to nothing less than a new kind of algebra in which algebraic symbols are replaced by braids and knots. First of all, given two algebras $B, C$ in a braided category (such as the representation of $U_{q}(\mathfrak{g})$ ) we have a braided tensor product $B \underline{\otimes} C$ algebra in the same category defined like a superalgebra but with -1 replaced by the braiding $\Psi_{C, B}$ : $C \otimes B \rightarrow B \otimes C$. Thus the tensor product becomes noncommutative (even if each algebra $B, C$ was commutative) - the two subalgebras 'commute' up to $\Psi$. This is the mathematical definition of braid statistics. In the braided line the joint algebra of the independent $x, y$ is $\mathbb{C}[x] \underline{\mathbb{Q}}[y]$ with $\Psi(x \otimes y)=q y \otimes x$. In the braided plane the braided tensor product is between one copy $x, y$ and the other $x^{\prime}, y^{\prime}$. The braiding $\Psi$ in this case is more complicated. In fact it is the same braiding from the $U_{q}\left(s u_{2}\right)$ spin $\frac{1}{2}$ representation that gave the Jones polynomial. The miracle that makes knot invariants is the same miracle that allows braided multilinear algebra and multivariable $q$-analysis.

The addition law in both the above examples makes them into braided groups [49]. They are like quantum groups or super-quantum groups but with braid statistics. Thus, there is a coproduct

$$
\Delta x=x \otimes 1+1 \otimes x, \quad \Delta y=y \otimes 1+1 \otimes y
$$

etc., (this is a more formal way to write $x+x^{\prime}, y+y^{\prime}$ ). But $\Delta: B \rightarrow B \underline{\otimes} B$ rather than mapping to the usual tensor product. We then write the braiding $\Psi$ as a braid crossing for 
(a)

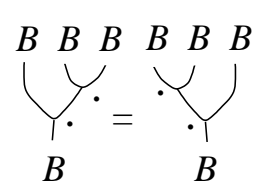

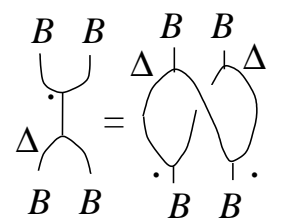

(b)

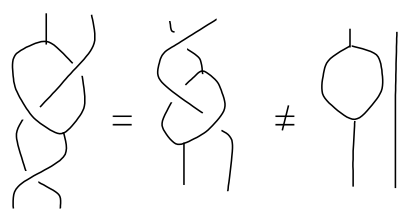

Figure 6: (a) Main axioms of a braided group as diagrams and (b) example of a braided calculation

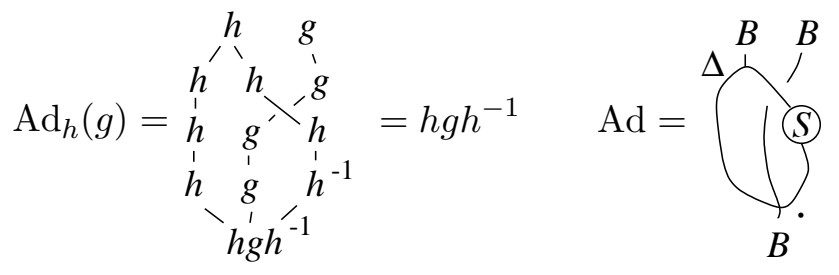

Figure 7: Conjugation written as a diagram

reasons explained in Section III.A. In this language one also draws the product $B \otimes B \rightarrow B$ as a map $Y$, the coproduct as $\lambda$, etc. Similarly with other maps, some strands coming in for the inputs and some leaving for the outputs. We then 'wire up' an algebraic expression by wiring outputs of one operation into the inputs of others, flowing down the page. When wires have to cross under or over, we have to chose one or the other as $\Psi$ or $\Psi^{-1}$. Algebra is then done as equalities of branched braids. For example, in Figure 6(a) we write the associativity of the product of an algebra and the homomorphism property of the coproduct $\Delta$ for a Hopf algebra or 'braided group' in this context. The coassociativity of the coproduct is the first part of Figure 6(a) turned up side down. There are also unity and antipode axioms. Part (b) of the figure is a example of a braided algebra calculation. Using this method one finds that all of the usual constructions for groups and quantum groups go through. For example, the notion of adjoint action or conjugation is shown in Figure 7. On the left is a 'breakdown' of the steps involved in usual conjugation in a group. One can think of a group trivially as a braided group with a coproduct that simply doubles up the group element. We double up $h$ in this way, move one of these past the $g$, apply the antipode or inversion operation $S$ and then multiply up. The corresponding diagram is shown on the right. In this way one arrives at a theory of algebras and groups which exists entirely at the level of braids and branches. One can do proofs, roughly speaking, by treating these as actual strings, i.e. this is a kind of knot-theoretic algebra. Fourier theory etc. all go through at this level.

Clearly such braided groups are, in particular, the correct foundation for $q$-deformed geometry based on $q$-planes and similar $q$-spaces. One of their main successes in the early 1990s was a 
more or less complete and systematic $q$-deformation of the main structures of special relativity and electromagnetism, i.e. $q$-Minkowski space and basic structures,

- $q$-Minkowski space as $2 \times 2$ braided Hermitian matrices

- $q$-addition etc., on $q$-Minkowski space

- $q$-Lorentz quantum group as a double cross product $\mathbb{C}_{q}\left[S U_{2}\right] \bowtie \mathbb{C}_{q}\left[S U_{2}\right]$

- $q$-Poincaré+scale quantum group $\mathbb{R}_{q}^{1,3} \nsucc \widetilde{U_{q}\left(\text { so } 1,3_{1,3}\right)}$

- $q$-partial derivatives

- $q$-differential forms

- $q$-epsilon tensor

- $q$-metric

- $q$-integration with Gaussian weight

- $q$-Fourier theory

- $q$-Green functions (but no closed form)

- $q$-* structures and $q$-Wick rotation

- $q$-conformal group $\mathbb{R}_{q}^{1,3} \rtimes \widetilde{U_{q}\left(s O_{1,3}\right)} \ltimes \mathbb{R}_{q}^{1,3}$

- $q$-diffeomorphism groups

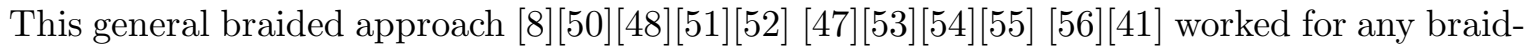
ing or 'R-matrix'. For example, the correct notion of 'braided matrices' $B(R)$ that goes along with the braided plane etc. above is generated by $u_{j}^{i}$ with the braided matrix relations

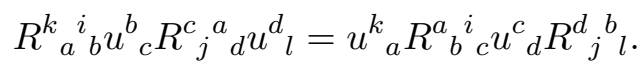

Just as the quantum matrices $A(R)$ in the previous section could be viewed as parameter-free versions of the algebra (87) but have their own life as geometric objects, these braided matrices $B(R)$ could also be viewed as a parameter-free version of the variants of (87) with two $R$ 's on each side. On the other hand all of their key properties, such as covariance under a background quantum group, the braid statistics and braided coproduct

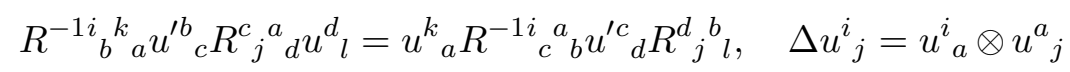


and so on, came out of the theory of braided groups as part of a close mathematical relationship called transmutation between $A(R)$ and $B(R)$ i.e. from the quantum to the braided versions. In fact this suggests a physical equivalence between the periodic and the open lattice systems by such transmutation that has not been explored much so far.

The specific choice of the same R-matrix as for the quantum plane or for $\mathbb{C}_{q}\left[S U_{2}\right]$ gives the algebra[8]

$$
\begin{gathered}
b a=q^{2} a b, \quad c a=q^{-2} a c, \quad d a=a d, \quad b c=c b+\left(1-q^{-2}\right) a(d-a), \\
d b=b d+\left(1-q^{-2}\right) a b, \quad c d=d c+\left(1-q^{-2}\right) c a
\end{gathered}
$$

of $q$-Minkowski space. This is the version with a central time $t=q^{-1} a+q d$ as promised. Similarly one can plug the standard R-matrix into general braided constructions and obtain the specific form of the other required $q$-algebras. In some cases this braided approach was confirmed by recovering algebras which had been proposed independently for this case by more ad hoc methods. In particular, the relations (111) were proposed as $q$-Minkowski space in [7] as an algebra on which the $q$-Lorentz group acts. Similarly Wess, Zumino et al. first proposed a large algebra with many generators and $q$-relations as $q$-Poincaré in [57. The braided approach provided R-matrix formulae, the braided addition and braided matrix structures of $q$-Minkowski space and the covariant action of $q$-Poincaré it. The latter came out of a general bosonisation theorem which asserts that for every braided group $B$ with background symmetry a (co)quasitriangular quantum group $H$ one has an equivalent ordinary (bosonic) quantum group $B \rtimes H$. The main thing is that from this communal effort a more or less definitive version of the mathematical structures for this particular toy model of spacetime is now known. In particular, one has $q$ Fourier transform to $q$-momentum space which turns out to be $q$-Minkowski space again (just like for $\mathbb{R}^{n}$ ). In the language of Section II it means that there are both 'quantum' and 'curved' aspects matching each other and isomorphic via the quantum metric. Using this one has no problem to define formal powerseries for the $q$-Green function as the inverse Fourier transform

$$
G_{q}(\vec{x})=\mathcal{F}^{-1}\left(\vec{p} \cdot \vec{p}-m^{2}\right)^{-1}
$$

so that, in principle, this is now defined.

There are some fundamental problems at the moment with this $q$-spacetime before one can expect real physical predictions (in contrast to the simpler model in Section II). First of all one does not generally have closed expressions such as for the $q$-Green functions above. The methods of $q$-analysis as in [46][58] are simply not yet far enough advanced to have nice names and properties for the kinds of powerseries functions encountered. This is a matter of time. Secondly, while the $q$-Poincaré coordinate algebra has a natural $*$-algebra structure so that 
one can study its representations in Hilbert spaces etc. (this was first done in the Euclidean case by G. Fiore) the $*$-structure does not respect the coproduct in the obvious way. One can understand this as rather fundamental to braided geometry for the following reason: when we deform classical constructions to braided ones we have to choose $\Psi$ or $\Psi^{-1}$ whenever wires cross. Sometimes neither will do, things get tangled up. But if we succeed it means that for every $q$-deformation there is another where we could have made the opposite choice in every case. So classical geometry bifurcates into two $q$-deformed geometries according to $\Psi$ or $\Psi^{-1}$. Moreover, the role of the $*$ operation is that it interchanges these two 54. Roughly speaking,

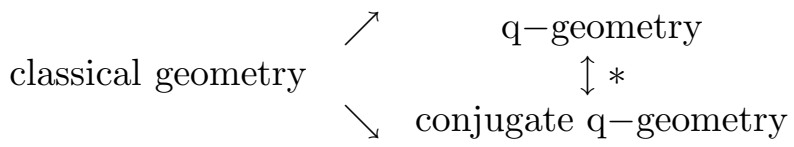

where the conjugate is constructed by interchanging the braiding with the inverse braiding (i.e. reversing braid crossings in the diagrammatic construction). For the simplest cases like the braided line it means interchanging $q, q^{-1}$. This is rather interesting given that the $*$-operation is a central foundation of quantum mechanics and our concepts of probability. But it also means one probably cannot do $q$-quantum mechanics etc., with $q$-geometry alone; one needs also the conjugate geometry.

Although there are such difficulties which make it hard at the moment to assess the physical significance of this kind of $q$-deformation, there are some important motivations and one should expect that some of them will eventually be realised using some later version of our present efforts in this direction. First of all we have said that the true meaning of $q$ is that it generalises the -1 of fermionic statistics. That is why it is dimensionless. It is nothing other than a parameter in a mathematical structure (the braiding) in a generalisation of our usual concepts of algebra and geometry, going a step beyond supergeometry. It is very likely, if not clear, that $q$ a root of unity would therefore be the correct setting for the treatment of certain anyonic systems where particles of anyonic statistics should be found. Or conversely one should identify and study known models with $q$-symmetry at $q$ a root of unity from the point of view of identifying the modes with anyonic statistics. This is clear but should be elaborated further. $q$-deformed constructions should then help in understanding the geometry of such systems. And of course there is the original physical meaning where $q$ is related to an anisotropy such as that due to an external magnetic field.

Other than these, there are some potential long-term reasons to $q$-deform, particularly spacetime as above. Thus, in [10] it was proposed that since $q$ was dimensionless and somewhat 'orthogonal' to physics it should be an ideal parameter for regularising any quantum field theory. Since most constructions in physics $q$-deform, such a regularisation scheme is much less brutal than say dimensional or Pauli-Villars regularisation as it preserves symmetries as $q$-symmetries, the $q$-epsilon tensor etc. In this context it seems at first too good a regularisation. Something has 
to go wrong for anomalies to appear. For example, it would be interesting to see exactly how the axial anomaly appears in this regularisation approach. The main thing that the regularisation loses is that only the Poincaré+scale $q$-deforms (the two get mixed up) which means that only massless particles should be treated in the fist place. The massive case would break even this invariance, giving different results for the two. Pushing the problem into the scale generator also suggests that a much nicer treatment of the renormalisation group should be possible in this context. Again a lot of this must await more development of the tools of $q$-analysis. At any rate the result in [10] is that $q$-deformation does indeed regularise, turning some of the infinities from a Feynman loop integration into poles $(q-1)^{-1}$.

Also, $q$-deformation might be useful as a next-order approximation to the geometry coming out of a known or unknown theory of quantum gravity. Thus, as well as being a good regulator one can envisage (in view of our general ideas about noncommutativity and the Planck scale) that the actual world is in reality better described by $q \neq 1$ due to Planck scale effects. This was the original reason given in [10] for $q$-deforming the basic structures of physics. The UV cut-off provided by a 'foam-like structure of space time' would instead be provided by $q \neq 1$. Moreover, if this is so then $q$-deformed quantum field theory should also appear coming out of quantum gravity as an approximation one better than the usual. Such a theory would be massless according to the above remarks (because there is no $q$-Poincaré without the scale generator). Or at least particle masses would be small compared to the Planck mass. How the $q$-scale invariance breaks would then be a mechanism for mass generation.

Recently, it was argued [59] that since loop gravity is linked to the Wess-Zumino-NovikovWitten (WZNW) model, which is linked to $U_{q}\left(s u_{2}\right)$ (or some other quantum group), that indeed $q$-geometry should appear coming out of quantum-gravity with cosmological constant $\Lambda$. There is even provided a formula

$$
q=e^{\frac{2 \pi i}{2+k}}, \quad k=\frac{6 \pi}{G_{\text {Newton }}^{2} \Lambda} .
$$

If so then the many tools of $q$-deformation developed in the 1990s would suddenly be applicable to study the next-to-classical structure of quantum-gravity. The fact that loop variable and spin-network methods 'tap into' the revolutions that have taken place in the last decade around quantum groups, knot theory and the WZNW model makes such a conjecture reasonable.

Whether it is a matter of $q$-regularisation of flat space or of actual Planck scale effects, there are several new things that happen for $q \neq 1$ which are not visible for $q=1$. Their physical meaning can only be guessed at but whatever it is, it should be deep.

- $q$-Minkowski space has two classical limits, related by duality. One is the commutative coordinates on $\mathbb{R}^{1,3}$ but there is another as the homogenized enveloping algebra $U\left(s u_{2} \oplus\right.$ $u(1))$. 
- $q$-Minkowski space 'quantises' a Poisson-bracket on $\mathbb{R}^{1,3}$ given by the action of the special conformal translations.

- When $q \neq 1$ this action of special conformal transformations is the braided group adjoint action of $q$-Minkowski space on itself as an additive braided group.

This first item is a version of the general result that the braided group versions (by transmutation) of the enveloping algebras $U_{q}(\mathfrak{g})$ and their $q$-coordinate algebras are isomorphic. That is, there is essentially only one object in $q$-geometry with different scaling limits as $q \rightarrow 1$ to give either the classical enveloping algebra of $\mathfrak{g}$ or the coordinate algebra of $G$. These self-duality isomorphisms involve dividing by $q-1$ and are therefore singular when $q=1$, i.e. this is totally alien to conventional geometric ideas. A homogenized enveloping algebra just means with relations $\xi \eta-\eta \xi=C[\xi, \eta]$ where the right hand side is the Lie bracket and $C$ is a central element. In the $q$-deformed case there is a similar $C=a d-q^{2} c b$ which is the $q$-Minkowski length; the mass-shell hyperboloid of $q$-Minkowski space is essentially the same algebra as $U_{q}\left(s u_{2}\right)$,

$$
\begin{gathered}
\mathbb{C}[\text { mass shell }] \stackrel{1 \leftarrow q}{\longleftarrow}\left(\mathbb{R}_{q}^{1,3} / C=1\right) \cong U_{q}\left(s u_{2}\right) \stackrel{q \rightarrow 1}{\longrightarrow} U\left(s u_{2}\right) \\
\left(\begin{array}{ll}
a & b \\
c & d
\end{array}\right) \cong\left(\begin{array}{cc}
q^{H} & q^{-\frac{1}{2}}\left(q-q^{-1}\right) q^{\frac{H}{2}} X_{-} \\
q^{-\frac{1}{2}}\left(q-q^{-1}\right) X_{+} q^{\frac{H}{2}} & q^{-H}+q^{-1}\left(q-q^{-1}\right)^{2} X_{+} X_{-}
\end{array}\right)
\end{gathered}
$$

which is a $q$-geometrical point of view on previously known 'matrix generators' 40] for quantum groups such as $U_{q}\left(s u_{2}\right)$ - in our case it comes $q$-geometrically from a covering isomorphism of $\mathbb{R}_{q}^{1,3}$ with the braided enveloping algebra $U\left(g l_{q, 2}\right)$ of a braided-Lie algebra $g l_{q, 2}$. Axioms and a general theory of such Lie algebra objects underlying quantum groups was one of the important technical achievements of braided groups in the mid 1990s [60]. Notice also that the two different scaling limits $q \rightarrow 1$ exactly implement the Fourier duality between noncommutative position and constrained momentum discussed in Section II.A.

Finally, we note that probably the greatest significance in the long term of the braided approach is that it solves the following 'uniformity of quantisation problem',

- There is only one universe. How do we know when we have quantised this or that space separately that they are consistent and fit together to a single quantum universe?

The braided approach solves this because we do not start with the Poisson brackets - they come from classicalisation - but from the deeper principle of braid statistics. Apart from giving the $q$-deformation of most structures in physics, it does it uniformly and in a generally consistent way because what what we deform is actually the category of vector spaces into a braided category. All constructions based on linear maps then deform coherently and consistently with 
each other as braid diagram constructions (so long as they do not get tangled). After that one inserts the formulae for specific braidings (e.g. generated by specific quantum groups) to get the $q$-deformation formulae. After that one semiclassicalises by taking commutators to lowest order, to get the Poisson-bracket that we have just quantised. Moreover, different quantum groups $U_{q}(\mathfrak{g})$ are all mutually consistent being related to each other by the inductive construction 36] mentioned at the end of Section III.A. We have seen this with the $q$-Lorentz and $q$-conformal groups for $q$-Minkowski space above.

\section{Quantum manifolds}

In this section we take a closer look at the progress towards the more general noncommutative differential geometry of which quanutm groups and braided groups should be a part. If one means by differential geometry 'bundles', 'connections', gauge theory etc then such a theory did emerged in the early 1990s in the work of T. Brzezinski and the author, with the by-now standard example of the $q$-monopole. Leading from this there is today a more or less complete theory that includes most of the naturally occurring examples but is a general theory not limited to special examples and models, i.e. has the same degree of 'flabbiness' as conventional geometry.

There are open problems so it should not be thought that this section represents the last word; the subject is still evolving but there is now something on the table. Among other things, our constructions are purely algebraic with operator and $C^{*}$-algebra considerations not fully worked. On the other hand, there is plenty of concrete motivation. As well as what has already been said, let us note that as a bonus this programme of noncommutative geometry will include discrete geometry as a special case. It gives a systematic way to do geometry on lattices, for example, somewhat different from existing ad hoc methods. This is depicted in Figure 8. Thus, the idea is to find a general algebraic notion of geometry that includes usual commutative coordinate algebras as a special case and that includes the kind of natural $q$-deformation and other examples coming out of quantum groups. The latter are a good testing ground because they have a parameter which we can set $q \rightarrow 1$ to verify the correct classical limit, i.e. we maintain 'eye-contact' with conventional geometry (this is not true of more abstract approaches based on $C^{*}$-algebras, for example). Next, when we are satisfied that we have the natural definitions we can specialise to finite-dimensional algebras. For example even commutative ones, which would be differential geometry on finite sets.

- If one can do all of geometry on finite sets then functional integrals etc. become finitedimensional integrals etc. and we can analytically compute the vacuum of $\mathrm{QCD}$, quantum gravity etc. in the discrete model.

- We can apply the formalism to finite (commutative or noncommutative) algebras to provide 


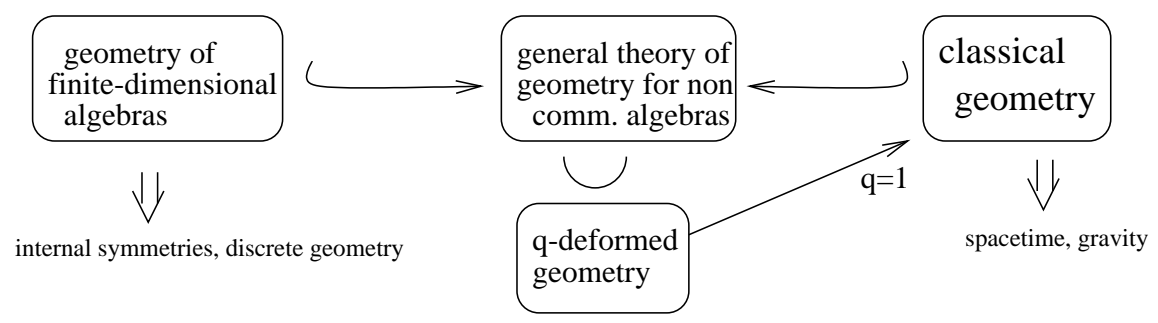

Figure 8: Noncommutative geometry found with the help of $q$-deformations can then specialise to finite-dimensional algebras

natural Dirac operators on quaternions $\mathbb{H}$ etc. which can figure in the internal structure of Lagrangians.

For example, Connes and J. Lott at the start of the 1990s proposed a way to noncommutativegeometrically package the contents of the standard model in a kind of Kaluza-Klein manner by working with the coordinate algebra of usual spacetime tensored with a finite-dimensional algebra $\mathbb{C} \oplus \mathbb{H}$. In other words, instead of a compact internal symmetry group one has a noncommutative space. One can build a 'Dirac' operator on the tensor product from one on the usual spacetime and one on the finite-dimensional algebra. The latter encodes the mass matrix and related phenomenological aspects of the theory. This was a novel approach which gave, in particular, a nice way to think about the Higgs field mexican-hat potential. On the other hand, from an abstract point of view almost any matrix can be taken as the 'Dirac' operator on the finite-dimensional algebra so one does not really get theoretical predictions for the details of that. The latter can only come from knowing more of the infrastructure of noncommutative differential geometry to determine what is the geometrically natural operator to take as Dirac on $\mathbb{C} \oplus \mathbb{H}$. While particular applications like this remain to be developed in the near future, it is clear that for either of the above reasons the general noncommutative differential geometry, even specialised to finite-dimensional algebras, can translate very directly into physical predictions.

\section{IV.A Quantum differential forms}

We begin here with the first of the different 'layers' of differential geometry, namely the notion of differential structure itself. Since we omit $C^{*}$ algebra considerations our coordinate algebra can be practically any (possibly noncommutative) algebra $M$. To specify the differential structure we in effect choose the cotangent space or differential 1-forms $\Omega^{1}$. Since one can multiply forms by 'functions' from the left and right, this should be an $M$-bimodule. There should also be a linear map d : $M \rightarrow \Omega^{1}$ such that

$$
\mathrm{d}(a b)=(\mathrm{d} a) b+a \mathrm{~d} b, \quad \forall a, b \in M
$$


and $\Omega^{1}$ should be spanned by elements of the form $a \mathrm{~d} b$. The main difference from what one might naively take here is that we do not assume that the left and right multiplications by $M$ coincide. For if $a \mathrm{~d} b=(\mathrm{d} b) a$ for all $a, b$, we would have $\mathrm{d}[a, b]=0$, which would not be at all suitable for a generic noncommutative algebra. Differential structures are not unique even classically, and even more non-unique in the quantum case. There is, however, one universal example of which others are quotients. This is

$$
\Omega_{\mathrm{univ}}^{1}=\mathrm{ker} \cdot \subset M \otimes M, \quad \mathrm{~d} a=a \otimes 1-1 \otimes a .
$$

The universal calculus was studied by algebraic topologists in the 1970s and is common to practically all approaches to noncommutative geometry. Our first task is to choose an appropriate quotient.

Classically, we do not think about this much because on a group there is a unique translationinvariant differential calculus; since we generally work with manifolds built on or closely related to groups we tend to take the inherited differential structure without thinking. In the quantum case, i.e. when $M$ is a quantum group one has a similar notion: a differential calculus is bicovariant if there are coactions $\Omega^{1} \rightarrow \Omega^{1} \otimes M, \Omega^{1} \rightarrow M \otimes \Omega^{1}$ forming a bicomodule and compatible with the bimodule structures and d. This natural definition was proposed by Woronowicz 61 at the end of the 1980s along with a couple of examples, but it took another decade before systematic classification results for the possible such calculi appeared [62]. By now the complete range of possibilities for all main classes of Hopf algebras are more or less understood. We begin with a sample of the general results, taken from 63] 62] by the author.

For the one-dimensional case of polynomials $M=k[x]$ with values in any field $k$, the coirreducible calculi (those with no further quotients) have the form $\Omega^{1}=k_{\lambda}[x]$ where $k_{\lambda}$ is a field extension of the form $k[\lambda]$ modulo $m(\lambda)=0$ and $m$ is an irreducible monic polynomial. The differential and bimodule structures are

$$
\mathrm{d} f(x)=\frac{f(x+\lambda)-f(x)}{\lambda}, \quad f(x) \cdot g(\lambda, x)=f(x+\lambda) g(\lambda, x), \quad g(\lambda, x) \cdot f(x)=g(\lambda, x) f(x)
$$

for functions $f$ and one-forms $g$. For example, the calculi on $\mathbb{C}[x]$ are classified by $\lambda_{0} \in \mathbb{C}$ (here $\left.m(\lambda)=\lambda-\lambda_{0}\right)$ and one has

$$
\Omega^{1}=\mathrm{d} x \mathbb{C}[x], \quad \mathrm{d} f=\mathrm{d} x \frac{f\left(x+\lambda_{0}\right)-f(x)}{\lambda_{0}}, \quad x \mathrm{~d} x=(\mathrm{d} x) x+\lambda_{0} .
$$

We see that the Newtonian case $\lambda_{0}=0$ is only one special point in the moduli space of quantum differential calculi. But if Newton had not supposed that differentials and forms commute he would have had no need to take this limit. What one finds with noncommutative geometry is that there is no need to take this limit at all. In particular, noncommutative geometry extends 
our usual concepts of geometry to lattice theory without taking the limit of the lattice spacing going to zero. It is also interesting that the most important field extension in physics, $\mathbb{R} \subset \mathbb{C}$, can be viewed noncommutative-geometrically with complex functions $\mathbb{C}[x]$ the quantum 1-forms on the algebra of real functions $\mathbb{R}[x]$.

We can similarly consider functions $M=\mathbb{C}[G]$ on a finite group. Then the coirreducible calculi correspond to nontrivial conjugacy classes $\mathcal{C} \subset G$ and have the form

$$
\Omega^{1}=\mathcal{C} \cdot \mathbb{C}[G], \quad \mathrm{d} f=\sum_{g \in \mathcal{C}} g \cdot\left(L_{g}(f)-f\right), \quad f \cdot g=g \cdot L_{g}(f)
$$

where $L_{g}(f)=f(g \cdot)$ is the translate of $f$. The cases of $\mathbb{C}[x]$ and $\mathbb{C}[G]$ are trivial enough to have been observed by hand before the general classification theorems arrived.

Next, for true examples of noncommutative coordinates we can consider $M=\mathbb{C} G$ generated by a finite group. It is the dual of the $\mathbb{C}[G]$ case but regarded 'up side down' as a noncommutative space when $G$ is nonAbelian. One has that the coirreducible calculi correspond to pairs $(V, \rho, \lambda)$ where $(V, \rho)$ is a nontrivial irreducible representation and $\lambda \in V / \mathbb{C}$. They have the form

$$
\Omega^{1}=V \cdot \mathbb{C} G, \quad \mathrm{~d} g=((\rho(g)-1) \lambda) \cdot g, \quad g \cdot v=(\rho(g) v) \cdot g
$$

where $g \in G$ is regarded as a 'function'. For $M=U(\mathfrak{g})$ as in Section II one has a similar construction for any irreducible representation $V$ of the Lie algebra $\mathfrak{g}$ and choice of ray $\lambda$ in it. The complete classification in the Lie cases of either the enveloping or the coordinate algebra are not known.

This covers the classical objects or their duals. For a finite-group bicrossproduct $\mathbb{C}[M] \backsim \mathbb{C} G$ the classification is a mixture of the two cases above and is given in [64]. Another method covers the Planck scale quantum group $\mathbb{C}[x] \bowtie \mathbb{C}[p]$ in Section II, namely this is a twisting by a cocycle of its classical limit $\mathbb{C}[\mathbb{R} \propto \mathbb{R}]$ and there is a theorem that its differentials are thereby in correspondence with those in the classical limit 21]. Finally, we come to the quantum groups like $\mathbb{C}_{q}[G]$ as in Section III. Here there is a theorem[62] that the coirreducible calculi are basically in correspondence with nontrivial irreducible representations $V$ of the Lie algebra and have the form

$$
\Omega^{1}=\left(V \otimes V^{*}\right) \cdot \mathbb{C}_{q}[G]
$$

In fact one has one natural calculus for each irreducible representation plus some 'shadows' or technical variants allowed according to the precise formulation of the relevant quantum groups and their duality (this is more a deficit in the technical definitions than anything else). For example, for $\mathbb{C}_{q}\left[S U_{2}\right]$ there is basically one bicovariant calculus for each spin $j$ with dimension $(2 j+1)^{2}$. The lowest is 4 dimensional. Actually the spanning vector space here is the 4dimensional braided-Lie algebra $g l_{q, 2}$ mentioned at the end of Section III.D. It is irreducible 
for generic $q$ but as $q \rightarrow 1$ it degenerates into $s u_{2} \oplus u(1)$. Correspondingly the 4-dimensional calculus becomes a direct sum of the usual 3-dimensional calculus on $S U_{2}$ and an additional operator, the Casimir operator or Laplacian:

- Bicovariant $\Omega^{1}$ for all main classes of quantum groups have been classified

- In particular, when we $q$-deform $S U_{2}$ its usual differentials and its Laplacian are necessarily bound up in one coirreducible 4-dimensional bicovariant calculus.

Other non-bicovariant calculi are possible also, including a standard 3-dimensional left-covariant calculus on $\mathbb{C}_{q}\left[S U_{2}\right]$ known since the original work of Woronowicz.

Finally, on a quantum group there is a natural extension to higher order forms and in fact an entire exterior algebra once the bicovariant 1-forms have been chosen[61]. Other extensions are also possible. Given the extension, one has a quantum cohomology defined in the usual way as closed forms modulo exact ones. To close with one non-quantum group example, consider any actual manifold with a finite good cover $\left\{U_{i}\right\}_{i \in I}$. Instead of building geometric invariants on a manifold and studying them modulo diffeomorphisms we can use the methods above to first pass to the skeleton of the manifold defined by its open set structure and do differential geometry directly on this indexing set $I$. Thus we take $M=\mathbb{C}[I]$ which just means collections $\left\{f_{i} \in \mathbb{C}\right\}$. The universal $\Omega^{1}$ is just matrices $\left\{f_{i j}\right\}$ vanishing on the diagonal. We use the intersection data for the open sets to set some of these to zero. Similarly for higher forms. Thus 65

$$
\begin{gathered}
\Omega^{1}=\left\{f_{i j} \mid U_{i} \cap U_{j} \neq \emptyset\right\}, \quad \Omega^{2}=\left\{f_{i j k} \mid U_{i} \cap U_{j} \cap U_{k} \neq \emptyset\right\} \\
(\mathrm{d} f)_{i j}=f_{i}-f_{j}, \quad(\mathrm{~d} f)_{i j k}=f_{i j}-f_{i k}+f_{j k}
\end{gathered}
$$

and so on. Then one has that the quantum cohomology is just the additive Cech cohomology of the original manifold.

Apart from cohomology one can start to do gauge theory, at least with trivial bundles. At this level a ' $\mathrm{U}(1)$ ' gauge field is just a differential form $\alpha \in \Omega^{1}$ and its curvature is $F=\mathrm{d} \alpha+\alpha \wedge \alpha$, etc. A gauge transform is

$$
\alpha^{\gamma}=\gamma^{-1} \alpha \gamma+\gamma^{-1} \mathrm{~d} \gamma, \quad F^{\gamma}=\gamma^{-1} F \gamma
$$

for any invertible 'function' $\gamma \in M$, and so on. One can certainly obtain interesting results even when the base is classical (but the calculus is quantum). For the example associated to open sets one has that the zero curvature gauge fields modulo gauge transformations recovers again the first Cech cohomology, but now in a multiplicative form. Or by chosing a quantum calculus even on usual $\mathbb{R}^{n}$ it is clear that nonlinear and higher-derivative equations could be viewed as zero curvature ones. Solutions would typically then be provided by $\alpha=\gamma^{-1} \mathrm{~d} \gamma$ i.e., pure gauge. 
There have been some first efforts in this direction in the physics literature. It should be clear at least that noncommutative differentials have the potential to unify and make clearer a whole range of otherwise ad-hoc constructions ranging from group theory to number theory to lattice differentials and integrable systems.

\section{IV.B Bundles and connections}

The next layer of differential geometry is bundles, connections, etc. Usually in physics one needs only the local picture with trivial bundles in each open set - but for a general noncommutative algebra $M$ there may be no reasonable 'open sets' and one has therefore to develop the global picture from the start. We need nontrivial bundles to cover physics such as in the BohmAharanov effect, potential effects such as the monopole and also to cover homogeneous spaces and the frame bundles of general 'manifolds'. None of these could be understood without a global point of view. In particular, the next quantum spaces after quantum groups and quantum-braided planes are quanutm homogeneous spaces and examples such as the quantum sphere $\mathbb{C}_{q}\left[S^{2}\right]$ (actually a two-parameter family of them) were known already by the end of the 1980s [66]. The required noncommutative differential geometry to really understand them as bundles came a few years later in [67.

Also note that this is a different problem from going from $U(1)$ gauge theory to nonAbelian, but the two can be handled together, i.e. we want a general quantum group as gauge group. For trivial bundles we could just take a gauge field as $\alpha \in \Omega^{1} \otimes U_{q}(\mathfrak{g})$ for example and write down similar formulae to those at the end of the last section. A gauge transform is an invertible element $\gamma \in M \otimes U_{q}(\mathfrak{g})$ etc. For our global geometrical picture however, we need to think of the quantum group geometrically and work with $H$ viewed as more like $\mathbb{C}[G]$ or a $q$-coordinate algebra $\mathbb{C}_{q}[G]$ etc. This is the setting for the present section. Also, to keep things simple we give formulae only for the universal differential calculus but the general case is also covered by making suitable quotients.

Basically, a classical bundle has a free action of a group and a local triviality property. In our algebraic terms we need an algebra $P$ in the role of 'coordinate algebra of the total space of the bundle' and a coaction $\Delta_{R}: P \rightarrow P \otimes H$ of the quantum group $H$ such that the fixed subalgebra is $M$,

$$
M=P^{H}=\left\{p \in P \mid \Delta_{R} p=p \otimes 1\right\} .
$$

Freeness and local triviality are replaced by the requirement that

$$
0 \rightarrow P\left(\Omega^{1} M\right) P \rightarrow \Omega^{1} P \stackrel{\text { ver }}{\longrightarrow} P \otimes \operatorname{ker} \epsilon \rightarrow 0
$$

is exact, where ver $=(\cdot \otimes \mathrm{id}) \Delta_{R}$ plays the role of generator of the vertical vector fields corre-

sponding classically to the action of the group (for each element of $H^{*}$ it maps $\Omega^{1} P \rightarrow P$ like a 
vector field). Exactness on the left says that the one-forms $P\left(\Omega^{1} M\right) P$ lifted from the base are exactly the ones annihilated by the vertical vector fields.

One can then define a connection as an equivariant splitting

$$
\Omega^{1} P=P\left(\Omega^{1} M\right) P \oplus \text { complement }
$$

i.e. an equivariant projection $\Pi$ on $\Omega^{1} P$. One can show the required analogue of the usual theory, i.e. that such a projection corresponds to a connection form

$$
\omega: \operatorname{ker} \epsilon \rightarrow \Omega^{1} P, \quad \text { ver } \circ \omega=1 \otimes \text { id }
$$

where $\omega$ intertwines with the adjoint coaction of $H$ on itself. Finally, one can define associated bundles. If $V$ is a vector space on which $H$ coacts then we define the associated 'bundles' $E^{*}=(P \otimes V)^{H}$ and $E=\operatorname{hom}_{H}(V, P)$, the space of intertwiners. The two bundles should be viewed geometrically as 'sections' in classical geometry of bundles associated to $V$ and $V^{*}$. Given a suitable (strong) connection one has a covariant derivative

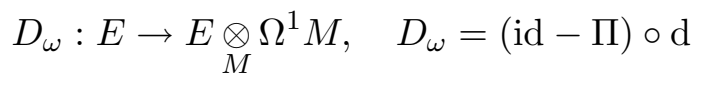

All of this can be checked out on the $q$-monopole bundle over the $q$-sphere 67. Recall that classically the inclusion $U(1) \subset S U_{2}$ in the diagonal has coset space $S^{2}$ and defines the $U(1)$ bundle over the sphere on which the monopole lives. The same idea works here, but since we deal with coordinate algebras the arrows are reversed. The coordinate algebra of $U(1)$ is the polynomials $\mathbb{C}\left[g, g^{-1}\right]$ and the classical inclusion becomes the projection

$$
\pi: \mathbb{C}_{q}\left[S U_{2}\right] \rightarrow \mathbb{C}\left[g, g^{-1}\right], \quad \pi\left(\begin{array}{ll}
a & b \\
c & d
\end{array}\right)=\left(\begin{array}{cc}
g & 0 \\
0 & g^{-1}
\end{array}\right)
$$

Its induced coaction $\Delta_{R}=(\mathrm{id} \otimes \pi) \Delta$ is by the degree defined as the number of $a, c$ minus the number of $b, d$ in an expression. The quantum sphere $\mathbb{C}_{q}\left[S^{2}\right]$ is the fixed subalgebra i.e. the degree zero part. Explicitly, it is generated by $b_{3}=a d, b_{+}=c d, b_{-}=a b$ with $q$-commutativity relations

$$
b_{ \pm} b_{3}=q^{ \pm 2} b_{3} b_{ \pm}+\left(1-q^{ \pm 2}\right) b_{ \pm}, \quad q^{2} b_{-} b_{+}=q^{-2} b_{+} b_{-}+\left(q-q^{-1}\right)\left(b_{3}-1\right)
$$

and the sphere equation $b_{3}^{2}=b_{3}+q b_{-} b_{+}$. When $q \rightarrow 1$ we can write $b_{ \pm}= \pm(x \pm \imath y), b_{3}=$ $z+\frac{1}{2}$ and the sphere equation becomes $x^{2}+y^{2}+z^{2}=\frac{1}{4}$ while the others become that $x, y, z$ commute. It turns out that we have a quantum bundle in the sense above and that there is a connection $\omega(g-1)=d \mathrm{~d} a-q b \mathrm{~d} c$ which, as $q \rightarrow 1$, becomes the usual Dirac monopole constructed algebraically.

It is easy to see that the 'matter fields' or sections of the associated vector bundles $E_{n}$ for each charge $n$ are just the degree $n$ parts of $\mathbb{C}_{q}\left[S U_{2}\right]$. The associated covariant derivative 
acts on these. This is also where the noncommutative differential geometry coming out of quantum groups links up with the more traditional $C^{*}$-algebra approach of $\mathrm{A}$. Connes and others. Traditionally a vector bundle over any algebra is defined as a finitely generated projective module. However, there was no notion of quantum principal bundle before quantum groups. The associated bundles $E_{n}$ for the $q$-monopole bundle indeed turned out to be finitely generated projective modules [68], i.e. there is an $(|n|+1) \times(|n|+1)$-matrix $e_{n}$ with values in $\mathbb{C}_{q}\left[S^{2}\right]$ with $e_{n}^{2}=e_{n}$ and $E_{n}=e_{n} \mathbb{C}_{q}\left[S^{2}\right]^{|n|+1}$. The covariant derivative for the monopole in these terms has the form $e_{n} \mathrm{~d} e_{n}$. For the lowest charge the projector is

$$
e_{1}=\left(\begin{array}{cc}
b_{3} & -q b_{-} \\
b_{+} & q^{2}\left(1-b_{3}\right)
\end{array}\right)
$$

The projectors are elements of the noncommutative $K$-theory $K_{0}\left(\mathbb{C}_{q}\left[S^{2}\right]\right)$ and have a duality pairing with Connes' cyclic cohomology 12] which for the $q$-monopole gives the correct answer as its Chern class. Thus the quantum groups approach ties up in the end with Connes' approach but provides more of the (so far algebraic) infrastructure of differential geometry - principal bundles, connection forms, etc. otherwise missing.

The potential applications of quantum group gauge theory hardly need to be elaborated. Among the more esoteric let us note that nonAbelian gauge fields provide invariants of manifolds and hence similarly one could obtain 'geometric' invariants of noncommutative algebras $M$. For example, for a classical manifold

$$
\left\{\begin{array}{c}
\text { Flat connections on } G-\text { bundle } \\
\text { modulo gauge }
\end{array}\right\} \cong \operatorname{hom}\left(\pi_{1}, G\right) / G
$$

using the holonomy. One can view this as a functor from groups to sets and the homotopy group $\pi_{1}$ as more or less the representing object in the category of groups. The same idea with quantum group gauge theory defines $\pi_{1}(M)$ as a homotopy quantum group for any algebra $M$ as more or less the representing object of the functor that assigns to a quantum group $H$ the set of zero-curvature gauge fields with this quantum structure group. This goes somewhat beyond vector bundles and $K$-theory alone. Although in principle defined, this idea has yet to be developed in a computable form. It is one of many directions for the future. Other directions include discrete e.g. finite models of QCD and functional integration in this setting.

Finally we mention that one needs to make a slight generalisation of the above to include other noncommutative examples of interest. In fact (and a little unexpectedly) the general theory above can be developed with only a coalgebra rather than a Hopf algebra $H$. Or dually it means only an algebra $A$ in place of the enveloping algebra of a Lie algebra. This was achieved more recently, in [69] [70], and allows us to include the full 2-parameter quantum spheres as well as (in principle) to all known $q$-deformed symmetric spaces. Beyond that, we can as mentioned apply the theory to our favourite finite-dimensional algebras or to commutative algebras with 
quantum differential calculi, etc. Again it seems likely that some startling applications along these lines will emerge in coming years.

\section{IV.C Quantum soldering forms and metrics}

We are finally ready to take the plunge and define a 'quantum manifold'. If our primary goal is to unify quantum theory and gravity through some noncommutative generalisation of geometry then the following at least puts something on the table to try out. This theory was only recently proposed in [71] and has therefore been little explored so far. But it does already predict a slight generalisation even of conventional Riemannian geometry as naturally appearing by semiclassicalisation. One could use conventional geometric methods to first explore the classical predictions of that generalisation even before getting into the noncommutative theory. The approach we take is basically that of a vierbein or, in global terms, a soldering form. This expresses gravity as a gauge theory of the frame bundle so that we can use the formalism of the previous section.

The first step is to define a generalised frame bundle or frame resolution of our algebra $M$ as a quantum principal bundle $\left(P, H, \Delta_{R}\right)$ over $M$, a comodule $V$ and an equivariant 'soldering form' $\theta: V \rightarrow P \Omega^{1} M \subset \Omega^{1} P$ such that the induced map

$$
E^{*} \rightarrow \Omega^{1} M, \quad p \otimes v \mapsto p \theta(v)
$$

is an isomorphism. What this does is to express the cotangent bundle as associated to a principal one. Other tensors are then similarly associated, for example vector fields are $E \cong \Omega^{-1} M$. Of course, all of this has to be done with suitable choices of differential calculi on $M, P, H$ whereas we have been focusing for simplicity on the universal calculi. There are some technicalities here but more or less the same definitions work in general. The working definition[71] of a quantum manifold is simply this data $\left(M, \Omega^{1}, P, H, \Delta_{R}, V, \theta\right)$. The definition works in that one has analogues of many usual results. For example, a connection $\omega$ on the frame bundle induces a covariant derivative $D_{\omega}$ on the associated bundle $E^{*}$ which maps over under the soldering isomorphism to a covariant derivative

$$
\nabla: \Omega^{1} M \rightarrow \Omega^{1} M \underset{M}{\otimes} \Omega^{1} M
$$

Its torsion is defined as corresponding similarly to $D_{\omega} \theta$.

Defining a Riemannian structure is harder. It turns out that it can be done in a 'self-dual' manner as follows. Given a framing, a 'generalised metric' isomorphism $\Omega^{-1} M \cong \Omega^{1} M$ between vector fields and one forms can be viewed as the existence of another framing $\theta^{*}: V^{*} \rightarrow\left(\Omega^{1} M\right) P$, which we call the coframing, this time with $V^{*}$. Nondegeneracy of the metric corresponds to $\theta^{*}$ inducing an isomorphism $E \cong \Omega^{1} M$. The working definition of a quantum Riemannian manifold 
is therefore the data $\left(M, \Omega^{1}, P, H, \Delta_{R}, V, \theta, \theta^{*}\right)$, where we have a framing and at the same time $\left(M, \Omega^{1}, P, H, \Delta_{R}, V^{*}, \theta^{*}\right)$ is another framing. The associated quantum metric is

$$
g=\theta^{*}\left(f^{a}\right) \theta\left(e_{a}\right) \in \Omega^{1} M \underset{M}{\otimes} \Omega^{1} M
$$

where $\left\{e_{a}\right\}$ is a basis of $V$ and $\left\{f^{a}\right\}$ is a dual basis (c.f. the canonical element exp from Fourier theory in Section II.C).

Now, this self-dual formulation of 'metric' as framing and coframing is symmetric between the two. One could regard the coframing as the framing and vice versa. From our original point of view its torsion tensor corresponding to $D_{\omega} \theta^{*}$ is some other tensor, which we call the cotorsion tensor. This is a new concept which did not exist in conventional differential geometry. We then define a generalised Levi-Civita connection on a quantum Riemannian manifold as the $\nabla$ of a connection $\omega$ such that the torsion and cotorsion tensors both vanish. The Riemannian curvature of course corresponds to the curvature of $\omega$, which is $d \omega+\omega \wedge \omega$, via the soldering form. I would not say that the Ricci tensor and Einstein tensor are understood abstractly enough in this formalism but of course one can just write down the relevant contractions and proceed blindly.

This is about as far as the programme has come at present. It is known[71] that every quantum group with bicovariant calculus is a quantum manifold in this sense. And for quantum groups such as $\mathbb{C}_{q}\left[S U_{2}\right]$ there is an Ad-invariant non-degenerate braided Killing form on the braided-Lie algebra $g l_{q, 2}$ in [60] which provides a coframing from a framing - so that quantum groups such as $\mathbb{C}_{q}\left[S U_{2}\right]$ with such differential calculi are quantum Riemannian manifolds in the required sense. At least with the universal calculus every quantum homogeneous space is a quantum manifold too. That includes quantum spheres, quantum planes etc. In fact, there is a notion of comeasuring or automorphism quantum group 41] for practically any algebra $M$ and when this has an antipode (which typically requires some form of completion) one can write $M$ as a quantum homogeneous space. So any $M$ is more or less a quantum manifold for some principal bundle (at least rather formally). This is analogous to the idea that any manifold is, rather formally, a homogeneous space of diffeomorphisms modulo diffeomorphisms fixing a base point. So the formalism does appear to be 'flabby' or general enough to sensibly write down field equations etc.

Finally, to get the physical meaning of the cotorsion tensor and other ideas coming out of this noncommutative Riemannian geometry, let us consider the semiclassical limit. What we find is that noncommutative geometry forces us to slightly generalise conventional Riemannian geometry itself. If noncommutative geometry is closer to what comes out of quantum gravity then this generalisation of conventional Riemannian geometry should be needed to include Planck scale effects or at least to be consistent with them when they emerge at the next order of approximation. The generalisation, more or less forced by the noncommutativity, is as follows: 
- We have to allow any group $G$ in the 'frame bundle', hence the more general concept of a 'frame resolution' ( $\left.P, G, V, \theta_{\mu}^{a}\right)$ or generalised manifold.

- The generalised metric $g_{\mu \nu}=\theta_{\mu}^{* a} \theta_{\nu a}$ corresponding to a coframing $\theta_{\mu}^{* a}$ is nondegenerate but need not be symmetric.

- The generalised Levi-Civita connection defined as having vanishing torsion and vanishing cotorsion respects the metric only in a skew sense

$$
\nabla_{\mu} g_{\nu \rho}-\nabla_{\nu} g_{\mu \rho}=0
$$

- The group $G$ is not unique (different flavours of frames are possible, e.g. an $E_{6}$-resolved manifold), not necessarily based on $S O_{n}$. This gives different flavours of covariant derivative $\nabla$ that can be induced by a connection form $\omega$.

- Even when $G$ is fixed and $g_{\mu \nu}$ is fixed, the generalised Levi-Civita condition does not fix $\nabla$ uniquely, i.e. one should use a first order $\left(g_{\mu \nu}, \nabla\right)$ or $\left(\theta, \theta^{*}, \omega\right)$ formalism.

To explain (138) we should note the general result 771 that for any generalised metric one has

$$
\nabla_{\mu} g_{\nu \rho}-\nabla_{\nu} g_{\mu \rho}=\text { CoTorsion }_{\mu \nu \rho}-\text { Torsion }_{\mu \nu \rho}
$$

where we use the metric to lower all indices.

This generalisation of Riemannian geometry includes special cases of symplectic geometry, where the generalised metric is totally antisymmetric. So the two are unified in our formulation, which is what we would expect if the theory is to be the semiclassicalisation of a theory unifying quantum theory and geometry. It is also remarkable that metrics with antisymmetric part are exactly what are needed in string theory to establish T-duality. In summary, one has on the table a general noncommutative Riemannian geometry to play with. One can try it out on simple examples such as quaternions or on discrete spaces (for example doing a functional integral as a finite-dimensional integral to do quantum gravity). This is a direction for ongoing work at present. One can also explore the duality ideas of Section II. In particular the observable-state duality should translate into a relation between gravity and thermodynamical entropy in this algebraic setting. Finally, it is at least clear that there are immediate predictions even at the classical level in terms of a classical generalisation of Riemannian geometry with antisymmetric parts to the metric, something with can be explored using conventional geometric methods. This is more or less the state of the art at the time of writing. While I doubt that these are anything more than first exploratory efforts, it does seem that something like this has to be a step in the right direction for the unification of quantum theory and gravity. 


\section{A new philosophical foundation for the next millennium}

Since the millennium happens only once in a thousand years, this may now be a good time to sit back and think a bit about the long long term implications (if any) of what we are doing. After all, lay persons turn to physicists for insights into the nature of physical reality. Apart from technical calculations, do we have anything to really tell them? While I doubt that quantum groups and noncommutative geometry are the end of the line - i.e. even more powerful concepts will be needed later on, they do demonstrate some general ideas which I will try to explain here. These more general ideas are, in a nutshell, about the nature of the relationship between mathematics and physics. It seems clear to me that, on top of technical advancements, a future revolution in our understanding of Nature will probably also need new philosophical input and so we should not shy away from thinking about that. I have saved this best part for the last, not least because it is of necessity very much my personal view even more than previous sections. Most of it was eventually published in my 1987 essay on the nature of physical reality[1] and it may be considered as background motivation for almost all of my own work since then.

A safe starting point should be that whatever we may say today about fundamental physics that is based on our past experience and not on the deepest philosophical principles is not likely to be correct or to survive very far into the next millennium other than as an approximation. So as a basis we should stick only to some of the deepest principles. In my opinion one of the deepest principles concerns the nature of mathematics itself. Namely, throughout mathematics one finds an intrinsic dualism between observer and observed as follows. When we think of a function $f$ being evaluated on $x \in X$, we could equally-well think of the same numbers as $x$ being evaluated on $f$ a member of some dual structure $f \in \hat{X}$ :

$$
\text { Result }=f(x)=x(f) .
$$

Such a 'turning of the tables' is a mathematical fact. For any mathematical concept $X$ one may consider maps or 'representations' from it to some self-evident class of objects (say rational numbers or for convenience real or complex numbers) wherein our results of measurements are

deemed to lie. Such representations themselves form a dual structure $\hat{X}$ of which elements of $X$ can be equally well viewed as representations. But is such a dual structure equally real? It was postulated in [1] that indeed this should be so in a complete theory,

- The search for a complete theory of physics is the search for a self-dual formulation in the above representation-theoretic sense (the principle of representation-theoretic self-duality)

Put another way, a complete theory of physics should admit a 'polarisation' into two halves each of which is the set of representations of the other. This division should be arbitrary - one should be able to reverse interpretations (or indeed consider canonical transformations to other choices of 'polarisation' if one takes the symplectic analogy). 
Note that by completeness here I do not mean knowing in more and more detail what is true in the real world. That consists of greater and greater complexity but it is not theoretical physics. I'm considering that a theorist wants to know why things are the way they are. Ideally I would like on my deathbed to be able to say that I have found the right point of view or theoretical-conceptual framework from which everything else follows. Working out the details of that would be far from trivial of course. We are taking at this point a more or less conventional reductionist viewpoint except that the Principle asserts that we will not have found the required point of view unless it is self-dual in the above sense.

We have already seen in Section II how such a general philosophy could translate in practice. In the setting of quantum gravity we take the view first of all that geometry - or 'gravity' is dual to quantum theory or matter. We discussed this for simple models such as spheres with constant curvature where it was achieved by Fourier theory. If we accept this then in general terms Planck scale physics has to unify these mutually dual concepts into one structure. Einstein's equation

$$
G_{\mu \nu} \propto T_{\mu \nu}
$$

may then even appear as some kind of self-duality equation within this self-dual context. Here the stress-energy tensor $T_{\mu \nu}$ relates to how matter responds to the geometry, while the Einstein tensor $G_{\mu \nu}$ measures how geometry responds to matter. This is the part of Mach's principle which apparently inspired Einstein. To do it properly one needs clearly some kind of noncommutative geometry because $T_{\mu \nu}$ should really be the quantum operator stress-energy and its coupling to $G_{\mu \nu}$ through its expectation value is surely only the first approximation or semiclassical limit of an operator version of (141). But an operator version of $G_{\mu \nu}$ only makes sense in the context of noncommutative geometry. What we would hope to find, in a suitable version of these ideas, is a self-dual setting where there is a dual interpretation in which $T_{\mu \nu}$ is the Einstein tensor of some dual system and $G_{\mu \nu}$ its stress-energy. In this way the duality and self-duality of the situation would be made manifest.

While the above remarks cannot yet be made fully precise at that level of generality, quantum groups provide a simple and soluble version of this unification problem. This is shown in Figure 9. Thus, the simplest theories of physics are based on Boolean algebras (a theory consists of classification of a 'universe' set into subsets); there is a well-known duality operation interchanging a subset and its complement. The next more advanced self-dual category is that of (locally compact) Abelian groups such as $\mathbb{R}^{n}$. In this case the set of 1-dimensional (ir)reps is again an Abelian group, i.e. the category of such objects is self-dual. In the topological setting one has $\hat{\mathbb{R}}^{n} \cong \mathbb{R}^{n}$ so that these groups (which are at the core of linear algebra) are self-dual objects in the self-dual category of Abelian groups. Of course, Fourier theory interchanges these two. More generally, to accommodate other phenomena we step away from the self-dual axis. Thus, nonAbelian Lie groups such as $S U_{2}$ as manifolds provide the simplest examples of curved 


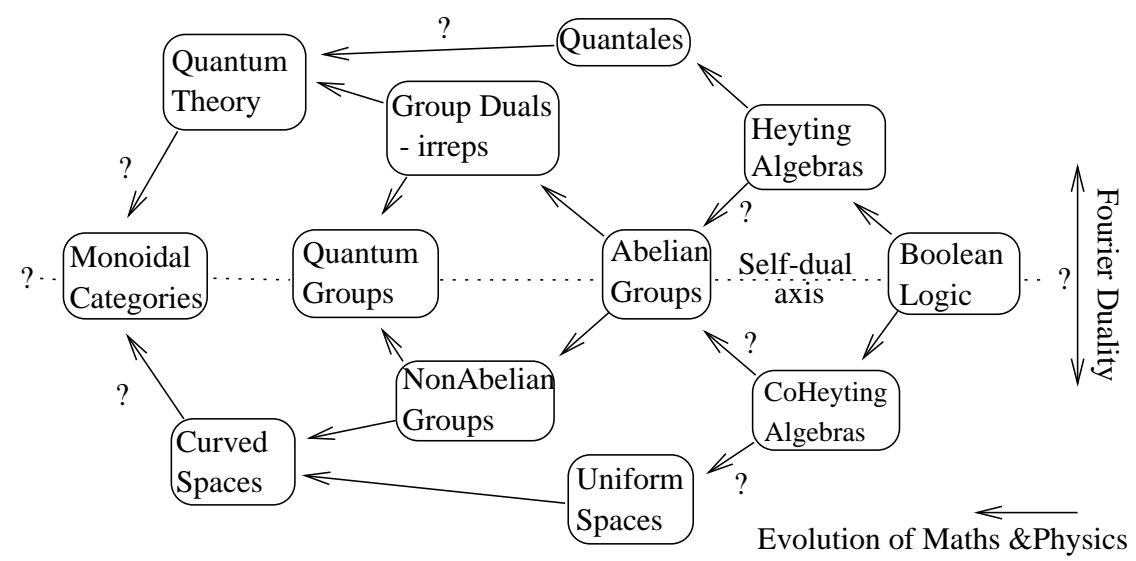

Figure 9: Representation-theoretic approach to Planck-scale physics. The unification of quantum and geometrical effects is a drive to the self-dual axis. Arrows denote inclusion functors

spaces. Their duals, which means constructing irreps, appear as central structures in quantum field theory (as judged by any course on particle physics in the 1960s). Wigner even defined a particle as an irrep of the Poincaré group. The unification of these two concepts, groups and groups duals was for many years an open problem in mathematics. Hopf algebras or quantum groups had already been invented in the 1940s and provided in particular the next more general self-dual category containing groups and group duals (or both coordinate algebras $\mathbb{C}[G]$ and enveloping algebras $U(\mathfrak{g})$ ) in which to attempt this unification. So the language existed but the problem to find examples of true quantum groups going genuinely beyond these and unifying them was open. It is remarkable that at the same time as this mathematical problem was going on, the problem of unifying quantum theory and gravity was going on in physics. Moreover, in a self-dual category we can even go further and look for self-dual objects as a further constraint on the detailed structure of the model. This was the thinking behind the bicrossproduct quantum groups in Section II.E[19]18]. We saw that the Planck-scale Hopf algebra indeed has both quantum and geometrical features and detailed structure, including dynamics not unlike a black-hole event horizon, coming out of the self-duality constraint. We also saw how the self-duality can be understood physically as an observable-state symmetry as in (140) between the geometry and the quantum aspects.

On the other hand the kind of general principle listed above is not just tied to this one setting. It could in principle both help predict the structure of more advanced theory of physics and, with hindsight, help us to conceptually organise its already know structure. This is because the structure of the theory of self-dual structures is nontrivial and not everything is possible. Knowing what is mathematically possible and combining with some postulates such as the above is not empty. For example, back in 1989 and motivated in the above manner it was shown that 
the category of monoidal categories (i.e. categories equipped with tensor products) was itself a self-dual category, i.e. that there was a construction $\hat{\mathcal{C}}$ for every such category $\mathcal{C}[72]$. Since then it has turned out that both conformal field theory and certain other quantum field theories can indeed be expressed in such categorical terms. Geometrical constructions can also be expressed categorically [73]. On the other hand, this categorical approach is still under-developed and its exact use and the exact nature of the required duality as a unification of quantum theory and gravity is still open. I would claim only 'something like that' (one should not expect too much from philosophy alone).

Another point to be made from Figure 9 is that if quantum theory and gravity already take us to very general structures such as categories themselves for the unifying concept then, in lay terms, what it means is that the required theory involves very general concepts indeed of a similar level to semiotics and linguistics (speaking about categories of categories etc.). It is almost impossible to conceive within existing mathematics (since it is itself founded in categories) what fundamentally more general structures would come after that. In other words, the required mathematics is running out it least in the manner that it was developed in this century (i.e. categorically) and at least in terms of the required higher levels of generality in which to look for self-dual structures. If the search for the ultimate theory of physics is to be restricted to logic and mathematics (which is surely what distinguishes science from, say, poetry), then this indeed correlates with our physical intuition that the unification of quantum theory and gravity is the last big unification for physics as we know it, or that theoretical physics as we know it is coming to an end. I would agree with this assertion except to say that the new theory will probably open up more questions which are currently considered metaphysics and make them physics, so I don't really think we will be out of a job even as theorists (and there will always be an infinite amount of 'what' work to be done even if the 'why' question was answered at some consensual level).

As well as seeking the 'end of physics', we can also ask more about its birth. Again there are many nontrivial and nonempty questions raised by the self-duality postulate. Certainly the key generalisation of Boolean logic to intuitionistic logic is to relax the axiom that $a \cup \tilde{a}=1$ (that $a$ or not $a$ is true). Such an algebra is called a Heyting algebra and can be regarded as the birth of quantum mechanics. Dual to this is the notion of a coHeyting algebra in which we relax the law that $a \cap \tilde{a}=0$. In such an algebra one can define the 'boundary' of a proposition as

$$
\partial a=a \cap \tilde{a}
$$

and show that it behaves like a derivation. This is surely the birth of geometry. How exactly this complementation duality extends to the Fourier duality for groups and on to the duality between more complex geometries and quantum theory is not completely understood, but there are conceptual 'physical' arguments that this should be so, put forward in [1]. Thus, in the 


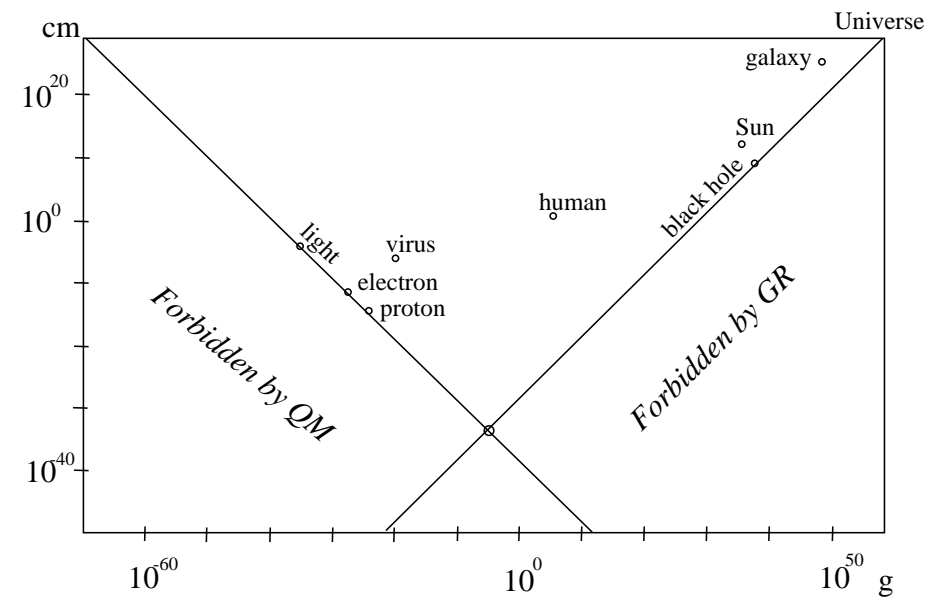

Figure 10: Range of physical phenomena, which lie in the wedge region with us in the middle. Log plots are mass-energy $\mathrm{v}$ size

simplest 'theories of physics' based only on logic one can work equally well with 'apples' or 'not-apples' as the names of subsets.

- What happens to this complementation duality in more advanced theories of physics? Apples curve space while not-apples do not, i.e. in physics one talks of apples as really existing while not-apples are merely an abstract concept.

Clearly the self-duality is lost in a theory of gravity alone. But we have argued[1] that when one considers both gravity and quantum theory, the self-duality can be restored. Thus when we say that a region is as full of apples as General Relativity allows (more matter simply forms a black hole which expands), which is the right hand limiting line in Figure 10, in the dual theory we might say that the region is as empty of not-apples as quantum theory allows, the limitation being the left slope in Figure 10. Here the uncertainty principle in the form of pair creation ensures that space cannot be totally empty of 'particles'. Although heuristic, these are arguments that quantum theory and gravity are dual and that this duality is an extension of complementation duality. Only a theory with both would be self-dual. Also, in view of a 'hole' moving in the opposite direction to a particle, the dual theory should also involves time reversal. The self-duality is something like CPT invariance but in a theory where gravitational and not only quantum effects are considered. We are proposing it as a key requirement for quantum-gravity. Diagrams similar to the right hand side of Figure 10 have been attributed to Brandon Carter as a tool to plot stellar evolution.

Note that as theoretical physicists we are not obliged to explain why the above should be a crude model for some of the structure of physics. We need only observe that it is a non-empty postulate with predictive and explanatory power. However, it is possible to speculate a little 
further and come to some philosophical conclusions. I will do this now. Why the principle of self-duality? Why such a central role for Fourier theory? The answer I believe is that something very general like this underlies the very nature of what it means to do science. The basic problem was proposed by Plato and comes under the heading of 'Plato's cave'. Namely, how can one tell the difference between reality and its representation or shadow. The modern answer according to [1] was that one should consider not one representation (or one angle of projection in Plato's cave) but all of them. Their collection is as much a valid mathematical structure as the original, as explained in above. Thus suppose that some theorist puts forward a theory in which there is an actual group $G$ say 'in reality' (this is where physics differs from mathematics) and some experimentalists construct tests of the theory and in so doing they routinely build representations or elements of $\hat{G}$. They will end up regarding $\hat{G}$ as 'real' and $G$ as merely an encoding of $\hat{G}$. The two points of view are in harmony because mathematically (in the appropriate context)

$$
G \cong \hat{\hat{G}}
$$

So far so good, but through the interaction and confusion between the experimental and theoretical points of view one will eventually have to consider both, i.e. $G \times \hat{G}$ as real. But then the theorists will come along and say that they don't like direct products, everything should interact with everything else, and will seek to unify $G, \hat{G}$ into some more complicated irreducible structure $G_{1}$, say. Then the experimentalists build $\hat{G}_{1} \ldots$ and so on. This is a kind of engine for the evolution of Science. For example, if one regarded, following Newton, that space $\mathbb{R}^{n}$ is real, its representations $\hat{\mathbb{R}}^{n}$ are derived quantities $\mathbf{p}=m \dot{\mathbf{x}}$. But after making diverse such representations one eventually regards both $\mathbf{x}$ and $\mathbf{p}$ as equally valid, equivalent via Fourier theory. But then we seek to unify them and introduce the CCR algebra (15). And so on. Note that this is not intended to be a historical account but a theory for how things could have gone in an ideal case without the twists and turns of human ignorance.

This is the plausibility reason that something like the principle of representation-theoretic self-duality should be observed. We have given arguments above that there is at least a correlation between the mathematical structure of self-dual structures and the progressive theories of physics from their birth in 'logic' to the projected forthcoming complete theory of everything. It should at least provide a guide to the properties that should be central in unknown theories of everything, such as what have become fashionable to call 'M-theory'. Now what if this kind of self-duality of structure was not only observed (in a crude form) but something like it, perhaps along with some other key postulates, actually fully characterised the structure of the allowed theories of physics? This is not out of the question given what we have said above about the level of generality already reached. It would be like giving a list of things that we expect from a complete theory - such as renormalisability, CPT-invariance, etc., except that we are considering such general versions of these 'constraints' that they are practically what it means to be 
a group of people following the scientific method. If this really pins down the ultimate theory then it would mean that:

- The ultimate theory of physics may be no more and no less than a self-discovery of the constraints in thinking that are taken on when one decides to look at the world as a physicist.

This is not at all the usual view of Nature as blindly 'out there' and is what I meant by a new philosophical foundation for theoretical physics. As big ideas go it is basically Kantian or Hegelian as opposed to the more conventional reductionist one that most physicists take for granted. The difference is that whereas Kant could only speculate, science backed by experiment, may actually be coming to the same conclusion in the not impossibly far future. It is important to note that this would not mean that physics is arbitrary or random any more than the different possible manifolds 'out there' are arbitrary. The space of all possible manifolds up to equivalence has a deep and rich structure and feels every bit as real to anyone who studies it; but it is a mathematical reality 'created' when we accept the axioms of a manifold. So what we are saying is that there is not such a fundamental difference between mathematical reality and physical reality. The main difference is that mathematicians are aware of the axioms while physicists tend to discover them 'backwards' by theorising from experience. I call this subjunctive point of view relative realism[1]. In it, we experience reality through choices that we have forgotten about at any given moment. If we become aware of the choice the reality it creates is dissolved or 'unconstructed'. On the other hand, the reader will say that the possibility of the theory of manifolds - that the game of manifold-hunting could have been played in the first place - is itself a reality, not arbitrary. It is, but at a higher level: it is a concrete fact in a more general theory of possible axiom systems of this type. To give another example, the reality of chess is created once we chose to play the game. If we are aware that it is a game, that reality is dissolved, but the rules of chess remain a reality although not within chess but within the space of possible board games. This gives a tree-like or hierarchical structure of reality. Reality is experienced as we look down the tree while 'awareness' or enlightenment is achieved as we look up the tree. When we are born we take on millions and millions of assumptions or rules through communication, which creates our day to day perception of reality, we then spend large parts of our lives questioning and attempting to unconstruct these assumptions as we seek understanding of the world. And from this perspective the fact that life appears somewhere near the middle of Figure 10, apart from the obvious explanation that phenomena become simpler as we approach the boundaries hence most complex in the middle so this is statistically where life would develop, has a different explanation: we created our picture of physical reality around ourselves and so not surprisingly we are near the middle. We may in effect have painted ourselves in a box by taking on certain assumptions about how to go about looking at the world. This would not be 
a bad thing but rather a statement about the origin of the laws of physics. I do doubt that it is ever going to be as simple as all that, but it is something to think about on a rainy day in the next millennium.

\section{Acknowledgments}

The author is a Reader and Royal Society University Research Fellow at QMW and a Senior Research Fellow during 1999-2001 at the Department of Applied Maths and Theoretical Physics, University of Cambridge, England, where some of the work was completed.

\section{References}

[1] S. Majid. Principle of representation-theoretic self-duality. Phys. Essays, 4(3):395-405, 1991.

[2] S. Majid. Beyond supersymmetry and quantum symmetry (an introduction to braided groups and braided matrices). In M-L. Ge and H.J. de Vega, editors, Quantum Groups, Integrable Statistical Models and Knot Theory, pages 231-282. World Sci., 1993.

[3] S. Majid. Introduction to braided geometry and q-Minkowski space. In L. Castellani and J. Wess, editors, Proceedings of the International School 'Enrico Fermi' CXXVII, pages 267-348. IOS Press, Amsterdam, 1996.

[4] V.G. Drinfeld. Quantum groups. In A. Gleason, editor, Proceedings of the ICM, pages 798-820, Rhode Island, 1987. AMS.

[5] M. Jimbo. A q-difference analog of $U(g)$ and the Yang-Baxter equation. Lett. Math. Phys., 10:63-69, 1985.

[6] S. Majid. Hopf algebras for physics at the Planck scale. J. Classical and Quantum Gravity, 5:1587-1606, 1988.

[7] U. Carow-Watamura, M. Schlieker, M. Scholl, and S. Watamura. Tensor representation of the quantum group $S L_{q}(2, \mathbb{C})$ and quantum Minkowski space. Z. Phys. C, 48:159, 1990.

[8] S. Majid. Examples of braided groups and braided matrices. J. Math. Phys., 32:3246-3253, 1991.

[9] G. Amelino-Camelia and S. Majid. Waves on noncommutative spacetime and gamma-ray bursts. Int. J. Mod. Phys. A, 2000 (in press).

[10] S. Majid. On q-regularization. Int. J. Mod. Phys. A, 5(24):4689-4696, 1990.

[11] S. Majid and H. Ruegg. Bicrossproduct structure of the $\kappa$-Poincaré group and noncommutative geometry. Phys. Lett. B, 334:348-354, 1994.

[12] A. Connes. Noncommutative Geometry. Academic Press, 1994. 
[13] A. Connes and M. Rieffel. Yang-mills theory over quantum tori. Contemp. Math., 62:237, 1987.

[14] S. Majid. Foundations of Quantum Group Theory. Cambridge Univeristy Press, 1995.

[15] S. Majid. Noncommutative geometry and quantum groups. Phil. Trans. Roy. Soc. A, 358:89-109, 2000.

[16] S. Majid. Meaning of noncommutative geometry and the Planck-scale quantum group. Springer Lec. Notes in Phys., 541:227-276, 2000.

[17] S. Majid. Duality principle and braided geometry. Springer Lec. Notes in Phys., 447:125$144,1995$.

[18] S. Majid. Physics for algebraists: Non-commutative and non-cocommutative Hopf algebras by a bicrossproduct construction. J. Algebra, 130:17-64, 1990.

[19] S. Majid. Non-commutative-geometric Groups by a Bicrossproduct Construction. PhD thesis, Harvard mathematical physics, 1988.

[20] O. Bratteli and D. W. Robinson. Operator Algebras and Quantum Statistical Mechanics, volume II. Springer-Verlag, 1979.

[21] S. Majid and R. Oeckl. Twisting of quantum differentials and the Planck scale Hopf algebra. Commun. Math. Phys., 205:617-655, 1999.

[22] G. Amelino-Camelia, J. Lukierski, and A. Nowicki. $\kappa$-deformed covariant phase space and quantum gravity uncertainty relations. Phys. Atom. Nucl., 61:1811-1815, 1988.

[23] S. Majid. Noncommutative geometric quantisation of photons and string field theory. Unpublished Harvard preprint, 1988.

[24] S. Majid. Fourier transforms on $\mathcal{A} / \mathcal{G}$ and knot invariants. J. Math. Phys., 32:924-927, 1990.

[25] S. Majid. C-statistical quantum groups and Weyl algebras. J. Math. Phys., 33:3431-3444, 1992.

[26] C. Klimcik and P. Severa. Dual non-abelian duality and the Drinfeld double. Phys. Lett. $B, 351: 455-462,1995$.

[27] E. Beggs and S. Majid. Poisson-lie T-duality for quasitriangular Lie bialgebras. Commun. Math. Phys., 2000 (in press).

[28] S. Majid. Matched pairs of Lie groups associated to solutions of the Yang-Baxter equations. Pac. J. Math., 141:311-332, 1990.

[29] S. Majid. Hopf-von Neumann algebra bicrossproducts, Kac algebra bicrossproducts, and the classical Yang-Baxter equations. J. Funct. Analysis, 95:291-319, 1991. 
[30] A. Connes and H. Moscovici. Hopf algebras, cyclic cohomology and the transverse index theory. Commun. Math. Phys., 198:199-246, 1998.

[31] A. Connes and D. Kreimer. Hopf algebras, renormalization and noncommutative geometry. Commun. Math. Phys., 199:203-242, 1998.

[32] L.D. Faddeev and L.A. Takhtajan. Hamiltonian Methods in the Theory of Solitons. Springer-Verlag, 1987.

[33] N.Yu. Reshetikhin and V.G. Turaev. Ribbon graphs and their invariants derived from quantum groups. Commun. Math. Phys., 127(1):1-26, 1990.

[34] V.F.R. Jones. Polynomial invariants of knots via von Neumann algebras. Bull. Amer. Math. Soc., 12:103-111, 1985.

[35] G. Lusztig. Introduction to Quantum groups. Birkhauser, 1993.

[36] S. Majid. Double bosonisation and the construction of $U_{q}(g)$. Math. Proc. Camb. Phil. Soc., 125:151-192, 1999.

[37] S. Majid. Braided-lie bialgebras. Pacific J. Math, 193:329-356, 2000.

[38] R.J. Baxter. Exactly Solvable Models in Statistical Mechanics. Academic, 1982.

[39] S.L. Woronowicz. Twisted $S U(2)$-group, an example of a non-commutative differential calculus. Publ. RIMS (Kyoto), 23:117-181, 1987.

[40] L.D. Faddeev, N.Yu. Reshetikhin, and L.A. Takhtajan. Quantization of Lie groups and Lie algebras. Leningrad Math. J., 1:193-225, 1990.

[41] S. Majid. Quantum and braided diffeomorphism groups. J. Geom. Phys., 28:94-128, 1998.

[42] V.G. Drinfeld. Hamiltonian structures on Lie groups, Lie bialgebras and the geometric meaning of the classical Yang-Baxter equations. Sov. Math. Dokl., 27:68, 1983.

[43] A.G. Reyman and M.A. Semenov-Tian-Shansky. Reduction of Hamiltonian systems, affine Lie algebras and Lax equations II. Invent. Math., 63:423-432, 1981.

[44] A. Macfarlane. On $q$-analogues of the quantum harmonic oscillator and the quantum group $S U(2)_{q}$. J. Phys. A, 22:4581, 1989.

[45] L.C. Biedenharn. The quantum group $S U_{q}(2)$ and the $q$-analogue of the boson operators. J. Phys. A, 22:L873, 1989.

[46] F.H. Jackson. On $q$-functions and a certain difference operator. Trans. Roy. Soc. Edin., 46:253-281, 1908.

[47] A. Kempf and S. Majid. Algebraic $q$-integration and Fourier theory on quantum and braided spaces. J. Math. Phys., 35:6802-6837, 1994.

[48] S. Majid. Braided momentum in the q-Poincaré group. J. Math. Phys., 34:2045-2058, 1993. 
[49] S. Majid. Braided groups. J. Pure and Applied Algebra, 86:187-221, 1993.

[50] S. Majid and U. Meyer. Braided matrix structure of $q$-Minkowski space and $q$-Poincaré group. Z. Phys. C, 63:357-362, 1994.

[51] S. Majid. Free braided differential calculus, braided binomial theorem and the braided exponential map. J. Math. Phys., 34:4843-4856, 1993.

[52] S. Majid. q-epsilon tensor for quantum and braided spaces. J. Math. Phys., 36:1991-2007, 1995.

[53] S. Majid. *-structures on braided spaces. J. Math. Phys., 36:4436-4449, 1995.

[54] S. Majid. Quasi-* structure on q-Poincaré algebras. J. Geom. Phys., 22:14-58, 1997.

[55] S. Majid. q-Euclidean space and quantum Wick rotation by twisting. J. Math. Phys., 35:5025-5034, 1994.

[56] S. Majid. Braided geometry of the conformal algebra. J. Math. Phys., 37:6495-6509, 1996.

[57] O. Ogievetsky, W.B. Schmidke, J. Wess, and B. Zumino. q-Deformed Poincaré algebra. Commun. Math. Phys., 150:495-518, 1992.

[58] T.H. Koornwinder. Orthogonal polynomials in connection with quantum groups. In P. Nevai, editor, Orthogonal Polynomials: Theory and Practice, number 294 in NATO ASI Series C, pages 257-292. Kluwer, 1990.

[59] S. Major and L. Smolin. Quantum deformation of quantum gravity. Nucl. Phys. B, 473:267290, 1996.

[60] S. Majid. Quantum and braided Lie algebras. J. Geom. Phys., 13:307-356, 1994.

[61] S.L. Woronowicz. Differential calculus on compact matrix pseudogroups (quantum groups). Commun. Math. Phys., 122:125-170, 1989.

[62] S. Majid. Classification of bicovariant differential calculi. J. Geom. Phys., 25:119-140, 1998.

[63] S. Majid. Quantum geometry of field extensions. J. Math. Phys., 40:2311-2323, 1999.

[64] E. Beggs and S. Majid. Quasitriangular and differential structures on bicrossproduct Hopf algebras. J. Algebra, 219:682-727, 1999.

[65] T. Brzeziński and S. Majid. Quantum differentials and the $q$-monopole revisited. Acta Appl. Math., 54:185-232, 1998.

[66] P. Podles. Quantum spheres. Lett. Math. Phys., 14:193-202, 1987.

[67] T. Brzeziński and S. Majid. Quantum group gauge theory on quantum spaces. Commun. Math. Phys., 157:591-638, 1993. Erratum 167:235, 1995.

[68] P. Hajac and S. Majid. Projective module description of the $q$-monopole. Commun. Math. Phys., 206:246-464, 1999. 
[69] T. Brzezinski and S. Majid. Coalgebra bundles. Commun. Math. Phys., 191, 1998. 467-492.

[70] T. Brzeziński and S. Majid. Quantum geometry of algebra factorisations and coalgebra bundles. Commun. Math. Phys., 2000 (in press).

[71] S. Majid. Quantum and braided group Riemannian geometry. J. Geom. Phys., 30:113-146, 1999.

[72] S. Majid. Representations, duals and quantum doubles of monoidal categories. Suppl. Rend. Circ. Mat. Palermo, Ser. II, 26:197-206, 1991.

[73] S. Majid. Some physical applications of category theory. Springer Lec. Notes in Phys., 375:131-142, 1991. 